\title{
Generalizations and refinements of
} three-tuple Diamond-Alpha integral Hölder's inequality on time scales

\author{
Fei Yan ${ }^{1 *}\left(\mathbb{D}\right.$ and Jianfeng Wang ${ }^{2}$
}

"Correspondence:
yanfei_ncepu@163.com
${ }^{1}$ North China Electric Power
University, Baoding, P.R. China
Full list of author information is
available at the end of the article

\begin{abstract}
In this paper, based on the existing Hölder's inequality, some new three-tuple diamond-alpha integral Hölder's inequalities on time scales are proposed and the related theorems and corollaries are given. At the same time, we also give the relevant conclusions and proof of $n$-tuple diamond-alpha integral Hölder's inequalities on time scales.
\end{abstract}

MSC: Primary 26D15; secondary 39A13

Keywords: Hölder's inequality; Diamond-Alpha integral; Three-tuple; Time scales; Minkowski's inequality

\section{Introduction}

Let $f(\delta)>0, g(\delta)>0, p>1,1 / p+1 / q=1$. If $f(\delta)$ and $g(\delta)$ are continuous real-valued functions on $[\xi, \sigma]$, then

$$
\int_{\xi}^{\sigma} f(\delta) g(\delta) d \delta \leq\left(\int_{\xi}^{\sigma} f^{p}(x) d \delta\right)^{1 / p}\left(\int_{\xi}^{\sigma} g^{q}(x) d \delta\right)^{1 / q}
$$

This famous Hölder inequality is extended in article [1] to the diamond- $\alpha$ integral Hölder inequality on time scales, in the following form:

Let $f, g, h:[\xi, \sigma] \rightarrow \mathbb{R}$ be $\diamond_{\alpha}$-integrable functions, and $1 / p+1 / q=1$ with $p>1$, then

$$
\int_{\xi}^{\sigma}|h(\delta)||f(\delta) g(\delta)| \nabla_{\alpha} \delta \leq\left(\int_{\xi}^{\sigma}|h(\delta)||f(\delta)|^{p} \diamond_{\alpha} \delta\right)^{\frac{1}{p}}\left(\int_{\xi}^{\sigma}|h(\delta)||g(\delta)|^{q} \diamond_{\alpha} \delta\right)^{\frac{1}{q}} .
$$

Since Hilger [2] proposed the time-scale theory in 1998, many researchers [3, 4] have made extensive promotions and applications of his theory. The classical analytic inequality [5-8], especially Hölder's inequality, plays a very important role in modern mathematics. Due to the importance of both, more and more scholars [9-15] have studied the intersections of two inequalities. The purpose of this article is to derive some generalizations and refinements of the three-tuple diamond- $\alpha$ integral Hölder inequality on time scales. The

(c) The Author(s) 2019. This article is licensed under a Creative Commons Attribution 4.0 International License, which permits use, sharing, adaptation, distribution and reproduction in any medium or format, as long as you give appropriate credit to the original author(s) and the source, provide a link to the Creative Commons licence, and indicate if changes were made. The images or other third party material in this article are included in the article's Creative Commons licence, unless indicated otherwise in a credit line to the material. If material is not included in the article's Creative Commons licence and your intended use is not permitted by statutory regulation or exceeds the permitted use, you will need to obtain permission directly from the copyright holder. To view a copy of this licence, visit http://creativecommons.org/licenses/by/4.0/. 
relevant conclusions of the $n$-tuple diamond- $\alpha$ integral Hölder inequality on time scales are also given.

\section{Main lemmas}

Before the main results are given in this paper, we need to introduce the following lemmas, which are helpful for the results of this paper.

Lemma 2.1 ([16]) Let $\sum_{j=1}^{m} \frac{1}{p_{j}}=1, \lambda_{j} \geq 0(j=1,2, \ldots, m)$. Then

(1) for $p_{j}>1$, we have

$$
\prod_{j=1}^{m} \lambda_{j} \leq \sum_{j=1}^{m} \frac{\lambda_{j}^{p_{j}}}{p_{j}}
$$

(2) for $0<p_{m}<1, p_{j}<0(j=1,2, \ldots, m-1)$, we have

$$
\prod_{j=1}^{m} \lambda_{j} \geq \sum_{j=1}^{m} \frac{\lambda_{j}^{p_{j}}}{p_{j}} .
$$

Lemma 2.2 ([10]) Let $\mathbb{T}$ be a time scale, $a, b \in \mathbb{T}$ with $a<b$ and $\sum_{j=1}^{m} \frac{1}{p_{j}}=1$. If $f_{j}(\delta)>0$, and $f_{j}(j=1,2, \ldots, m)$ is continuous real-valued function on $[\xi, \sigma]_{\mathbb{T}}$, then

(1) for $p_{j}>1$, we have

$$
\int_{\xi}^{\sigma} \prod_{j=1}^{m} f_{j}(\delta) \diamond_{\alpha} \delta \leq \prod_{j=1}^{m}\left(\int_{\xi}^{\sigma} f_{j}^{p_{j}}(\delta) \diamond_{\alpha} \delta\right)^{1 / p_{j}},
$$

(2) for $0<p_{m}<1, p_{j}<0(j=1,2, \ldots, m-1)$, we have

$$
\int_{\xi}^{\sigma} \prod_{j=1}^{m} f_{j}(\delta) \diamond_{\alpha} \delta \geq \prod_{j=1}^{m}\left(\int_{\xi}^{\sigma} f_{j}^{p_{j}}(\delta) \diamond_{\alpha} \delta\right)^{1 / p_{j}}
$$

Lemma $2.3([17])$ Let $f, g, h: \mathbb{T} \rightarrow \mathbb{R}$ be $\diamond$-integrable on $[\xi, \sigma]_{\mathbb{T}}, p>1$ with $q=p /(p-1)$. Then we have

$$
\int_{\xi}^{\sigma}|h(\delta)||f(\delta) g(\delta)| \nabla \delta \leq\left(\int_{\xi}^{\sigma}|h(\delta)||f(\delta)|^{p} \diamond \delta\right)^{1 / p}\left(\int_{\xi}^{\sigma}|h(\delta)||g(\delta)|^{q} \diamond \delta\right)^{1 / q} .
$$

Lemma 2.4 ([17]) Let $f, g, h: \mathbb{T} \rightarrow \mathbb{R}$ be $\diamond$-integrable on $[\xi, \sigma]_{\mathbb{T}}, p>1$ with $q=p /(p-1)$. Then we have

$$
\begin{aligned}
& \left(\int_{\xi}^{\sigma}|h(\delta)||f(\delta)+g(\delta)|^{p} \diamond \delta\right)^{1 / p} \\
& \quad \leq\left(\int_{\xi}^{\sigma}|h(\delta)||f(\delta)|^{p} \diamond \delta\right)^{1 / p}+\left(\int_{\xi}^{\sigma}|h(\delta)||g(\delta)|^{p} \diamond \delta\right)^{1 / p} .
\end{aligned}
$$

Lemma $2.5([17])$ Let $f, g, h: \mathbb{T} \rightarrow \mathbb{R}$ be $\diamond$-integrable on $[\xi, \sigma]_{\mathbb{T}}, 0<p<1$ with $q=p /(p-1)$. If $g^{q}$ is $\diamond$-integrable on $[\xi, \sigma]_{\mathbb{T}}$, then

$$
\int_{\xi}^{\sigma}|h(\delta)||f(\delta) g(\delta)| \diamond \delta \geq\left(\int_{\xi}^{\sigma}|h(\delta)||f(\delta)|^{p} \diamond \delta\right)^{1 / p}\left(\int_{\xi}^{\sigma}|h(\delta)||g(\delta)|^{q} \diamond \delta\right)^{1 / q} .
$$


Lemma 2.6 ([17]) Letf, $g, h: \mathbb{T} \rightarrow \mathbb{R}$ be $\diamond$-integrable on $[\xi, \sigma]_{\mathbb{T}}, 0<p<1$ with $q=p /(p-1)$.

Then we have

$$
\begin{aligned}
& \left(\int_{\xi}^{\sigma}|h(\delta)||f(\delta)+g(\delta)|^{p} \diamond \delta\right)^{1 / p} \\
& \quad \geq\left(\int_{\xi}^{\sigma}|h(\delta)||f(\delta)|^{p} \diamond \delta\right)^{1 / p}+\left(\int_{\xi}^{\sigma}|h(\delta)||g(\delta)|^{p} \diamond \delta\right)^{1 / p} .
\end{aligned}
$$

\section{Main results about diamond- $\alpha$ integral Hölder's inequality}

Now, based on Tian's $[18,19]$ research results, we will give the following generalizations and refinements of the three-tuple diamond- $\alpha$ integral and $n$-tuple diamond- $\alpha$ integral Hölder inequality on time scales.

Theorem 3.1 Let $\mathbb{T}$ be a time scale $a, b \in \mathbb{T}$ with $a<b$ and $\alpha_{k j} \in \mathbb{R}(j=1,2, \ldots, m, k=$ $1,2, \ldots, s), \sum_{k=1}^{s} \frac{1}{p_{k}}=1, \sum_{k=1}^{s} \alpha_{k j}=0$. If $f_{j}(\delta)>0$, and $f_{j}(j=1,2, \ldots, m)$ is a continuous realvalued function on $[\xi, \sigma]_{\mathbb{T}}$, then

(1) for $p_{k}>1$, we have

$$
\begin{aligned}
& \int_{\xi_{1}}^{\sigma_{1}} \int_{\xi_{2}}^{\sigma_{2}} \int_{\xi_{3}}^{\sigma_{3}} \prod_{j=1}^{m} f_{j}\left(\delta_{1}, \delta_{2}, \delta_{3}\right) \diamond_{\alpha} \delta_{1} \diamond_{\alpha} \delta_{2} \diamond_{\alpha} \delta_{3} \\
& \quad \leq \prod_{k=1}^{s}\left(\int_{\xi_{1}}^{\sigma_{1}} \int_{\xi_{2}}^{\sigma_{2}} \int_{\xi_{3}}^{\sigma_{3}} \prod_{j=1}^{m} f_{j}^{1+p_{k} \alpha_{k j}}\left(\delta_{1}, \delta_{2}, \delta_{3}\right) \diamond_{\alpha} \delta_{1} \diamond_{\alpha} \delta_{2} \diamond_{\alpha} \delta_{3}\right)^{\frac{1}{p_{k}}},
\end{aligned}
$$

(2) for $0<p_{s}<1, p_{k}<0(k=1,2, \ldots, s-1)$, we have

$$
\begin{aligned}
& \int_{\xi_{1}}^{\sigma_{1}} \int_{\xi_{2}}^{\sigma_{2}} \int_{\xi_{3}}^{\sigma_{3}} \prod_{j=1}^{m} f_{j}\left(\delta_{1}, \delta_{2}, \delta_{3}\right) \diamond_{\alpha} \delta_{1} \diamond_{\alpha} \delta_{2} \diamond_{\alpha} \delta_{3} \\
& \geq \prod_{k=1}^{s}\left(\int_{\xi_{1}}^{\sigma_{1}} \int_{\xi_{2}}^{\sigma_{2}} \int_{\xi_{3}}^{\sigma_{3}} \prod_{j=1}^{m} f_{j}^{1+p_{k} \alpha_{k j}}\left(\delta_{1}, \delta_{2}, \delta_{3}\right) \diamond_{\alpha} \delta_{1} \diamond_{\alpha} \delta_{2} \diamond_{\alpha} \delta_{3}\right)^{\frac{1}{p_{k}}} .
\end{aligned}
$$

Proof (1) Set

$$
g_{k}\left(\delta_{1}, \delta_{2}, \delta_{3}\right)=\left(\prod_{j=1}^{m} f_{j}^{1+p_{k} \alpha_{k j}}\left(\delta_{1}, \delta_{2}, \delta_{3}\right)\right)^{1 / p_{k}} .
$$

Applying the assumptions $\sum_{k=1}^{s} \frac{1}{p_{k}}=1$ and $\sum_{k=1}^{s} \alpha_{k j}=0$, by computing, we can observe that

$$
\begin{aligned}
& \prod_{k=1}^{s} g_{k}\left(\delta_{1}, \delta_{2}, \delta_{3}\right) \\
& \quad=g_{1} g_{2} \cdots g_{s} \\
& \quad=\left(\prod_{j=1}^{m} f_{j}^{1+a_{1} \alpha_{1 j}}\left(\delta_{1}, \delta_{2}, \delta_{3}\right)\right)^{1 / a_{1}}\left(\prod_{j=1}^{m} f_{j}^{1+a_{2} \alpha_{2 j}}\left(\delta_{1}, \delta_{2}, \delta_{3}\right)\right)^{1 / a_{2}} \cdots
\end{aligned}
$$




$$
\begin{aligned}
& \times\left(\prod_{j=1}^{m} f_{j}^{1+a_{s} \alpha_{s_{j}}}\left(\delta_{1}, \delta_{2}, \delta_{3}\right)\right)^{1 / a_{s}} \\
= & \prod_{j=1}^{m} f_{j}^{1 / a_{1}+\alpha_{1 j}}\left(\delta_{1}, \delta_{2}, \delta_{3}\right) \prod_{j=1}^{m} f_{j}^{1 / a_{2}+\alpha_{2 j}}\left(\delta_{1}, \delta_{2}, \delta_{3}\right) \cdots \\
& \times \prod_{j=1}^{m} f_{j}^{1 / a_{s}+\alpha_{s j}}\left(\delta_{1}, \delta_{2}, \delta_{3}\right) \\
= & \prod_{j=1}^{m} f_{j}^{1 / a_{1}+1 / a_{2}+\cdots+1 / a_{s}+\alpha_{1 j}+\alpha_{2 j}+\cdots+\alpha_{s j}}\left(\delta_{1}, \delta_{2}, \delta_{3}\right)=\prod_{j=1}^{m} f_{j}\left(\delta_{1}, \delta_{2}, \delta_{3}\right) .
\end{aligned}
$$

Hence, we obtain

$$
\begin{aligned}
& \int_{\xi_{1}}^{\sigma_{1}} \int_{\xi_{2}}^{\sigma_{2}} \int_{\xi_{3}}^{\sigma_{3}} \prod_{j=1}^{m} f_{j}\left(\delta_{1}, \delta_{2}, \delta_{3}\right) \diamond_{\alpha} \delta_{1} \diamond_{\alpha} \delta_{2} \diamond_{\alpha} \delta_{3} \\
& =\int_{\xi_{1}}^{\sigma_{1}} \int_{\xi_{2}}^{\sigma_{2}} \int_{\xi_{3}}^{\sigma_{3}} \prod_{k=1}^{s} g_{k}\left(\delta_{1}, \delta_{2}, \delta_{3}\right) \diamond_{\alpha} \delta_{1} \diamond_{\alpha} \delta_{2} \diamond_{\alpha} \delta_{3} .
\end{aligned}
$$

By the Hölder inequality (3), we find

$$
\begin{aligned}
& \int_{\xi_{1}}^{\sigma_{1}} \int_{\xi_{2}}^{\sigma_{2}} \int_{\xi_{3}}^{\sigma_{3}} \prod_{k=1}^{s} g_{k}\left(\delta_{1}, \delta_{2}, \delta_{3}\right) \diamond_{\alpha} \delta_{1} \diamond_{\alpha} \delta_{2} \diamond_{\alpha} \delta_{3} \\
& \quad \leq \prod_{k=1}^{s}\left(\int_{\xi_{1}}^{\sigma_{1}} \int_{\xi_{2}}^{\sigma_{2}} \int_{\xi_{3}}^{\sigma_{3}} g_{k}^{p_{k}}\left(\delta_{1}, \delta_{2}, \delta_{3}\right) \diamond_{\alpha} \delta_{1} \diamond_{\alpha} \delta_{2} \diamond_{\alpha} \delta_{3}\right)^{1 / p_{k}} .
\end{aligned}
$$

Substituting $g_{k}\left(\delta_{1}, \delta_{2}, \delta_{3}\right)$ into the inequality (12) can be obtained

$$
\begin{aligned}
& \int_{\xi_{1}}^{\sigma_{1}} \int_{\xi_{2}}^{\sigma_{2}} \int_{\xi_{3}}^{\sigma_{3}} \prod_{j=1}^{m} f_{j}\left(\delta_{1}, \delta_{2}, \delta_{3}\right) \diamond_{\alpha} \delta_{1} \diamond_{\alpha} \delta_{2} \diamond_{\alpha} \delta_{3} \\
& \leq \prod_{k=1}^{s}\left(\int_{\xi_{1}}^{\sigma_{1}} \int_{\xi_{2}}^{\sigma_{2}} \int_{\xi_{3}}^{\sigma_{3}} \prod_{j=1}^{m} f_{j}^{1+p_{k} \alpha_{k j}}\left(\delta_{1}, \delta_{2}, \delta_{3}\right) \diamond_{\alpha} \delta_{1} \diamond_{\alpha} \delta_{2} \diamond_{\alpha} \delta_{3}\right)^{\frac{1}{p_{k}}} .
\end{aligned}
$$

(2) After the same proof as inequality (9), we get

$$
\begin{aligned}
& \int_{\xi_{1}}^{\sigma_{1}} \int_{\xi_{2}}^{\sigma_{2}} \int_{\xi_{3}}^{\sigma_{3}} \prod_{k=1}^{s} g_{k}\left(\delta_{1}, \delta_{2}, \delta_{3}\right) \diamond_{\alpha} \delta_{1} \diamond_{\alpha} \delta_{2} \diamond_{\alpha} \delta_{3} \\
& \geq \prod_{k=1}^{s}\left(\int_{\xi_{1}}^{\sigma_{1}} \int_{\xi_{2}}^{\sigma_{2}} \int_{\xi_{3}}^{\sigma_{3}} g_{k}^{p_{k}}\left(\delta_{1}, \delta_{2}, \delta_{3}\right) \diamond_{\alpha} \delta_{1} \diamond_{\alpha} \delta_{2} \diamond_{\alpha} \delta_{3}\right)^{1 / p_{k}} .
\end{aligned}
$$

Substituting $g_{k}\left(\delta_{1}, \delta_{2} . \delta_{3}\right)$ into the $(12)$ can be obtained

$$
\int_{\xi_{1}}^{\sigma_{1}} \int_{\xi_{2}}^{\sigma_{2}} \int_{\xi_{3}}^{\sigma_{3}} \prod_{j=1}^{m} f_{j}\left(\delta_{1}, \delta_{2}, \delta_{3}\right) \diamond_{\alpha} \delta_{1} \diamond_{\alpha} \delta_{2} \diamond_{\alpha} \delta_{3}
$$




$$
\geq \prod_{k=1}^{s}\left(\int_{\xi_{1}}^{\sigma_{1}} \int_{\xi_{2}}^{\sigma_{2}} \int_{\xi_{3}}^{\sigma_{3}} \prod_{j=1}^{m} f_{j}^{1+p_{k} \alpha_{k j}}\left(\delta_{1}, \delta_{2}, \delta_{3}\right) \diamond_{\alpha} \delta_{1} \diamond_{\alpha} \delta_{2} \diamond_{\alpha} \delta_{3}\right)^{\frac{1}{p_{k}}} .
$$

The proof of Theorem 3.1 is accomplished.

Corollary 3.2 Under the conditions of Theorem 3.1, let $s=m, \alpha_{k j}=-t / p_{k}$ for $k \neq j$ and $\alpha_{j j}=t\left(1-1 / p_{j}\right)$ with $t \in \mathbb{R}$, then

(1) for $p_{k}>1$, we have the following inequality:

$$
\begin{aligned}
\int_{\xi_{1}}^{\sigma_{1}} & \int_{\xi_{2}}^{\sigma_{2}} \int_{\xi_{3}}^{\sigma_{3}} \prod_{j=1}^{m} f_{j}\left(\delta_{1}, \delta_{2}, \delta_{3}\right) \diamond_{\alpha} \delta_{1} \diamond_{\alpha} \delta_{2} \diamond_{\alpha} \delta_{3} \\
\leq & \prod_{k=1}^{m}\left(\int_{\xi_{1}}^{\sigma_{1}} \int_{\xi_{2}}^{\sigma_{2}} \int_{\xi_{3}}^{\sigma_{3}}\left(\prod_{j=1}^{m} f_{j}\left(\delta_{1}, \delta_{2}, \delta_{3}\right)\right)^{1-t}\right. \\
& \left.\times\left(f_{k}^{p_{k}}\left(\delta_{1}, \delta_{2}, \delta_{3}\right)\right)^{t} \diamond_{\alpha} \delta_{1} \diamond_{\alpha} \delta_{2} \diamond_{\alpha} \delta_{3}\right)^{1 / p_{k}}
\end{aligned}
$$

(2) $0<p_{m}<1, p_{k}<0(k=1,2, \ldots, m-1)$, we have the following reverse inequality:

$$
\begin{aligned}
\int_{\xi_{1}}^{\sigma_{1}} \int_{\xi_{2}}^{\sigma_{2}} \int_{\xi_{3}}^{\sigma_{3}} \prod_{j=1}^{m} f_{j}\left(\delta_{1}, \delta_{2}, \delta_{3}\right) \diamond_{\alpha} \delta_{1} \diamond_{\alpha} \delta_{2} \diamond_{\alpha} \delta_{3} \\
\geq \prod_{k=1}^{m}\left(\int_{\xi_{1}}^{\sigma_{1}} \int_{\xi_{2}}^{\sigma_{2}} \int_{\xi_{3}}^{\sigma_{3}}\left(\prod_{j=1}^{m} f_{j}\left(\delta_{1}, \delta_{2}, \delta_{3}\right)\right)^{1-t}\right. \\
\left.\quad \times\left(f_{k}^{p_{k}}\left(\delta_{1}, \delta_{2}, \delta_{3}\right)\right)^{t} \diamond_{\alpha} \delta_{1} \diamond_{\alpha} \delta_{2} \diamond_{\alpha} \delta_{3}\right)^{1 / p_{k}}
\end{aligned}
$$

On the basis of Theorem 3.1, we give the $n$-tuple diamond- $\alpha$ integral Hölder's inequality on time scales.

Theorem 3.3 Let $\mathbb{T}$ be a time scale $\xi, \sigma \in \mathbb{T}$ with $\xi<\sigma$ and $\alpha_{k j} \in \mathbb{R}(j=1,2, \ldots, m, k=$ $1,2, \ldots, s), \sum_{k=1}^{s} \frac{1}{p_{k}}=1, \sum_{k=1}^{s} \alpha_{k j}=0$. If $f_{j}(\delta)>0$, and $f_{j}(j=1,2, \ldots, m)$ is a continuous realvalued function on $[\xi, \sigma]_{\mathbb{T}}$, then

(1) for $p_{k}>1$, we have the following inequality:

$$
\begin{aligned}
& \int_{\xi_{1}}^{\sigma_{1}} \int_{\xi_{2}}^{\sigma_{2}} \cdots \int_{\xi_{n}}^{\sigma_{n}} \prod_{j=1}^{m} f_{j}\left(\delta_{1}, \delta_{2}, \ldots, \delta_{n}\right) \diamond_{\alpha} \delta_{1} \diamond_{\alpha} \delta_{2} \ldots \diamond_{\alpha} \delta_{n} \\
& \quad \leq \prod_{k=1}^{s}\left(\int_{\xi_{1}}^{\sigma_{1}} \int_{\xi_{2}}^{\sigma_{2}} \cdots \int_{\xi_{n}}^{\sigma_{n}} \prod_{j=1}^{m} f_{j}^{1+p_{k} \alpha_{k j}}\left(\delta_{1}, \delta_{2}, \ldots, \delta_{n}\right) \diamond_{\alpha} \delta_{1} \diamond_{\alpha} \delta_{2} \cdots \diamond_{\alpha} \delta_{n}\right)^{\frac{1}{p_{k}}},
\end{aligned}
$$

(2) for $0<p_{s}<1, p_{k}<0(k=1,2, \ldots, s-1)$, we have the following reverse inequality:

$$
\int_{\xi_{1}}^{\sigma_{1}} \int_{\xi_{2}}^{\sigma_{2}} \cdots \int_{\xi_{n}}^{\sigma_{n}} \prod_{j=1}^{m} f_{j}\left(\delta_{1}, \delta_{2}, \ldots, \delta_{n}\right) \diamond_{\alpha} \delta_{1} \diamond_{\alpha} \delta_{2} \cdots \diamond_{\alpha} \delta_{n}
$$




$$
\geq \prod_{k=1}^{s}\left(\int_{\xi_{1}}^{\sigma_{1}} \int_{\xi_{2}}^{\sigma_{2}} \cdots \int_{\xi_{n}}^{\sigma_{n}} \prod_{j=1}^{m} f_{j}^{1+p_{k} \alpha_{k j}}\left(\delta_{1}, \delta_{2}, \ldots, \delta_{n}\right) \diamond_{\alpha} \delta_{1} \diamond_{\alpha} \delta_{2} \ldots \diamond_{\alpha} \delta_{n}\right)^{\frac{1}{p_{k}}} .
$$

Proof Similar to the proof of Theorem 3.1, we get the result of Theorem 3.3.

Remark 3.4 The three-tuple diamond-alpha inequalities in Theorem 3.1 and the $n$-tuple diamond- $\alpha$ inequalities in Theorem 3.3 are generalizations to Theorem 3.3 in Ref. [10].

Theorem 3.5 Let $\mathbb{T}$ be a time scale, $\xi, \sigma \in \mathbb{T}$ with $\xi<\sigma$ and $r \in \mathbb{R}, \alpha_{k j} \in \mathbb{R}(j=$ $1,2, \ldots, m, k=1,2, \ldots, s), \sum_{k=1}^{s} \frac{1}{p_{k}}=r, \sum_{k=1}^{s} \alpha_{k j}=0$. If $f_{j}(\delta)>0$, and $f_{j}(j=1,2, \ldots, m)$ is a continuous real-valued function on $[\xi, \sigma]_{\mathbb{T}}$, then

(1) for $r p_{k}>1$, we have the following inequality:

$$
\begin{aligned}
& \int_{\xi_{1}}^{\sigma_{1}} \int_{\xi_{2}}^{\sigma_{2}} \int_{\xi_{3}}^{\sigma_{3}} \prod_{j=1}^{m} f_{j}\left(\delta_{1}, \delta_{2}, \delta_{3}\right) \diamond_{\alpha} \delta_{1} \diamond_{\alpha} \delta_{2} \diamond_{\alpha} \delta_{3} \\
& \quad \leq \prod_{k=1}^{s}\left(\int_{\xi_{1}}^{\sigma_{1}} \int_{\xi_{2}}^{\sigma_{2}} \int_{\xi_{3}}^{\sigma_{3}} \prod_{j=1}^{m} f_{j}^{1+r p_{k} \alpha_{k j}}\left(\delta_{1}, \delta_{2}, \delta_{3}\right) \diamond_{\alpha} \delta_{1} \diamond_{\alpha} \delta_{2} \diamond_{\alpha} \delta_{3}\right)^{\frac{1}{r p_{k}}},
\end{aligned}
$$

(2) for $0<r p_{k}<1, r p_{k}<0(k=1,2, \ldots, s-1)$, we have the following reverse inequality:

$$
\begin{aligned}
& \int_{\xi_{1}}^{\sigma_{1}} \int_{\xi_{2}}^{\sigma_{2}} \int_{\xi_{3}}^{\sigma_{3}} \prod_{j=1}^{m} f_{j}\left(\delta_{1}, \delta_{2}, \delta_{3}\right) \diamond_{\alpha} \delta_{1} \diamond_{\alpha} \delta_{2} \diamond_{\alpha} \delta_{3} \\
& \geq \prod_{k=1}^{s}\left(\int_{\xi_{1}}^{\sigma_{1}} \int_{\xi_{2}}^{\sigma_{2}} \int_{\xi_{3}}^{\sigma_{3}} \prod_{j=1}^{m} f_{j}^{1+r p_{k} \alpha_{k j}}\left(\delta_{1}, \delta_{2}, \delta_{3}\right) \diamond_{\alpha} \delta_{1} \diamond_{\alpha} \delta_{2} \diamond_{\alpha} \delta_{3}\right)^{\frac{1}{r p_{k}}} .
\end{aligned}
$$

Proof (1) According to $r p_{k}>1$ and $\sum_{k=1}^{s} \frac{1}{p_{k}}=r$, we get $\sum_{k=1}^{s} \frac{1}{r p_{k}}=1$. Then, by inequality (9), we immediately obtain the inequality (18).

(2) According to $0<r p_{k}<1, r p_{k}<0(k=1,2, \ldots, s-1)$ and $\sum_{k=1}^{s} \frac{1}{p_{k}}=r$, we have $\sum_{k=1}^{s} \frac{1}{r p_{k}}=1$, by inequality (10), we immediately have the inequality (19). This completes the proof.

Similarly, on the basis of Theorem 3.5, we give the $n$-tuple diamond- $\alpha$ integral Hölder's inequality on time scales.

Theorem 3.6 Let $\mathbb{T}$ be a time scale, $\xi, \sigma \in \mathbb{T}$ with $\xi<\sigma$ and $r \in \mathbb{R}, \alpha_{k j} \in \mathbb{R}(j=$ $1,2, \ldots, m, k=1,2, \ldots, s), \sum_{k=1}^{s} \frac{1}{p_{k}}=r, \sum_{k=1}^{s} \alpha_{k j}=0$. If $f_{j}(\delta)>0$, and $f_{j}(j=1,2, \ldots, m)$ is a continuous real-valued function on $[\xi, \sigma]_{\mathbb{T}}$, then

(1) for $r p_{k}>1$, we have the following inequality:

$$
\begin{aligned}
& \int_{\xi_{1}}^{\sigma_{1}} \int_{\xi_{2}}^{\sigma_{2}} \ldots \int_{\xi_{n}}^{\sigma_{n}} \prod_{j=1}^{m} f_{j}\left(\delta_{1}, \delta_{2}, \ldots, \delta_{n}\right) \diamond_{\alpha} \delta_{1} \diamond_{\alpha} \delta_{2} \cdots \diamond_{\alpha} \delta_{n} \\
& \quad \leq \prod_{k=1}^{s}\left(\int_{\xi_{1}}^{\sigma_{1}} \int_{\xi_{2}}^{\sigma_{2}} \cdots \int_{\xi_{n}}^{\sigma_{n}} \prod_{j=1}^{m} f_{j}^{1+r p_{k} \alpha_{k j}}\left(\delta_{1}, \delta_{2}, \ldots, \delta_{n}\right) \diamond_{\alpha} \delta_{1} \diamond_{\alpha} \delta_{2} \ldots \diamond_{\alpha} \delta_{n}\right)^{\frac{1}{r p_{k}}},
\end{aligned}
$$


(2) for $0<r p_{k}<1, r p_{k}<0(k=1,2, \ldots, s-1)$, we have the following reverse inequality:

$$
\begin{aligned}
& \int_{\xi_{1}}^{\sigma_{1}} \int_{\xi_{2}}^{\sigma_{2}} \cdots \int_{\xi_{n}}^{\sigma_{n}} \prod_{j=1}^{m} f_{j}\left(\delta_{1}, \delta_{2}, \ldots, \delta_{n}\right) \diamond_{\alpha} \delta_{1} \diamond_{\alpha} \delta_{2} \cdots \diamond_{\alpha} \delta_{n} \\
& \geq \prod_{k=1}^{s}\left(\int_{\xi_{1}}^{\sigma_{1}} \int_{\xi_{2}}^{\sigma_{2}} \cdots \int_{\xi_{n}}^{\sigma_{n}} \prod_{j=1}^{m} f_{j}^{1+r p_{k} \alpha_{k j}}\left(\delta_{1}, \delta_{2}, \ldots, \delta_{n}\right) \diamond_{\alpha} \delta_{1} \diamond_{\alpha} \delta_{2} \cdots \diamond_{\alpha} \delta_{n}\right)^{\frac{1}{r p_{k}}}
\end{aligned}
$$

Proof Similar to the proof of Theorem 3.5, we get the result of Theorem 3.6.

Remark 3.7 For the inequality of Theorem 3.4 in the Reference [10], we put forward Theorem 3.5 and Theorem 3.6 as the generalization results.

Theorem 3.8 Assume that $\mathbb{T}$ is a time scale, $\xi, \sigma \in \mathbb{T}$ with $\xi<\sigma$ and $p_{k}>0, \alpha_{k j} \in \mathbb{R}(j=$ $1,2, \ldots, m, k=1,2, \ldots, s), \sum_{k=1}^{s} \frac{1}{p_{k}}=1, \sum_{k=1}^{s} \alpha_{k j}=0, f_{j}, h: \mathbb{T} \rightarrow \mathbb{R}$. If $h$ and $f_{j}$ are $\diamond$-integrable on $[\xi, \sigma]_{\mathbb{T}}$, then the following assertions hold true.

(1) For $p_{k}>1$, one has

$$
\begin{aligned}
& \int_{\xi_{1}}^{\sigma_{1}} \int_{\xi_{2}}^{\sigma_{2}} \int_{\xi_{3}}^{\sigma_{3}}\left|h\left(\delta_{1}, \delta_{2}, \delta_{3}\right)\right|\left|\prod_{j=1}^{m} f_{j}\left(\delta_{1}, \delta_{2}, \delta_{3}\right)\right| \diamond \delta_{1} \diamond \delta_{2} \diamond \delta_{3} \\
& \leq \prod_{k=1}^{s}\left(\int_{\xi_{1}}^{\sigma_{1}} \int_{\xi_{2}}^{\sigma_{2}} \int_{\xi_{3}}^{\sigma_{3}}\left|h\left(\delta_{1}, \delta_{2}, \delta_{3}\right)\right| \prod_{j=1}^{m}\left|f_{j}\left(\delta_{1}, \delta_{2}, \delta_{3}\right)\right|^{1+p_{k} \alpha_{k j}} \diamond \delta_{1} \diamond \delta_{2} \diamond \delta_{3}\right)^{1 / p_{k}}
\end{aligned}
$$

(2) For $0<p_{s}<1, p_{k}<0(k=1,2, \ldots, s-1), f_{j}^{1+p_{k} \alpha_{k j}}$ is $\diamond$-integrable on $[\xi, \sigma]_{\mathbb{T}}$, one has

$$
\begin{aligned}
& \int_{\xi_{1}}^{\sigma_{1}} \int_{\xi_{2}}^{\sigma_{2}} \int_{\xi_{3}}^{\sigma_{3}}\left|h\left(\delta_{1}, \delta_{2}, \delta_{3}\right)\right|\left|\prod_{j=1}^{m} f_{j}\left(\delta_{1}, \delta_{2}, \delta_{3}\right)\right| \diamond \delta_{1} \diamond \delta_{2} \diamond \delta_{3} \\
& \geq \prod_{k=1}^{s}\left(\int_{\xi_{1}}^{\sigma_{1}} \int_{\xi_{2}}^{\sigma_{2}} \int_{\xi_{3}}^{\sigma_{3}}\left|h\left(\delta_{1}, \delta_{2}, \delta_{3}\right)\right| \prod_{j=1}^{m}\left|f_{j}\left(\delta_{1}, \delta_{2}, \delta_{3}\right)\right|^{1+p_{k} \alpha_{k j}} \diamond \delta_{1} \diamond \delta_{2} \diamond \delta_{3}\right)^{1 / p_{k}}
\end{aligned}
$$

Proof (1) Let

$$
g_{k}\left(\delta_{1}, \delta_{2}, \delta_{3}\right)=\left(\prod_{j=1}^{m} f_{j}^{1+p_{k} \alpha_{k j}\left(\delta_{1}, \delta_{2}, \delta_{3}\right)}\right)^{1 / p_{k}}
$$

Based on the assumptions $\sum_{k=1}^{s} \frac{1}{p_{k}}=1$ and $\sum_{k=1}^{s} \alpha_{k j}=0$, from a direct computation, it is obvious to show that

$$
\begin{aligned}
& \prod_{k=1}^{s} g_{k}\left(\delta_{1}, \delta_{2}, \delta_{3}\right) \\
& \quad=g_{1} g_{2} \cdots g_{s} \\
& \quad=\left(\prod_{j=1}^{m} f_{j}^{1+a_{1} \alpha_{1 j}}\left(\delta_{1}, \delta_{2}, \delta_{3}\right)\right)^{1 / a_{1}}\left(\prod_{j=1}^{m} f_{j}^{1+a_{2} \alpha_{2 j}}\left(\delta_{1}, \delta_{2}, \delta_{3}\right)\right)^{1 / a_{2}} \ldots
\end{aligned}
$$




$$
\begin{aligned}
& \times\left(\prod_{j=1}^{m} f_{j}^{1+a_{s} \alpha_{s_{j}}}\left(\delta_{1}, \delta_{2}, \delta_{3}\right)\right)^{1 / a_{s}} \\
= & \prod_{j=1}^{m} f_{j}^{1 / a_{1}+\alpha_{1 j}}\left(\delta_{1}, \delta_{2}, \delta_{3}\right) \prod_{j=1}^{m} f_{j}^{1 / a_{2}+\alpha_{2 j}}\left(\delta_{1}, \delta_{2}, \delta_{3}\right) \cdots \\
& \times \prod_{j=1}^{m} f_{j}^{1 / a_{s}+\alpha_{s j}}\left(\delta_{1}, \delta_{2}, \delta_{3}\right) \\
= & \prod_{j=1}^{m} f_{j}^{1 / a_{1}+1 / a_{2}+\cdots+1 / a_{s}+\alpha_{1 j}+\alpha_{2 j}+\cdots+\alpha_{s j}}\left(\delta_{1}, \delta_{2}, \delta_{3}\right)=\prod_{j=1}^{m} f_{j}\left(\delta_{1}, \delta_{2}, \delta_{3}\right) .
\end{aligned}
$$

From the above result, we can obtain

$$
\prod_{k=1}^{s} g_{k}\left(\delta_{1}, \delta_{2}, \delta_{3}\right)=\prod_{j=1}^{m} f_{j}\left(\delta_{1}, \delta_{2}, \delta_{3}\right) .
$$

Hence, we have

$$
\begin{gathered}
\int_{\xi_{1}}^{\sigma_{1}} \int_{\xi_{2}}^{\sigma_{2}} \int_{\xi_{3}}^{\sigma_{3}}\left|h\left(\delta_{1}, \delta_{2}, \delta_{3}\right)\right|\left|\prod_{j=1}^{m} f_{j}\left(\delta_{1}, \delta_{2}, \delta_{3}\right)\right| \diamond \delta_{1} \diamond \delta_{2} \diamond \delta_{3} \\
=\int_{\xi_{1}}^{\sigma_{1}} \int_{\xi_{2}}^{\sigma_{2}} \int_{\xi_{3}}^{\sigma_{3}}\left|h\left(\delta_{1}, \delta_{2}, \delta_{3}\right)\right|\left|\prod_{j=1}^{m} g_{k}\left(\delta_{1}, \delta_{2}, \delta_{3}\right)\right| \diamond \delta_{1} \diamond \delta_{2} \diamond \delta_{3} .
\end{gathered}
$$

It follows from Hölder's inequality (5) that

$$
\begin{aligned}
& \int_{\xi_{1}}^{\sigma_{1}} \int_{\xi_{2}}^{\sigma_{2}} \int_{\xi_{3}}^{\sigma_{3}}\left|h\left(\delta_{1}, \delta_{2}, \delta_{3}\right)\right|\left|\prod_{j=1}^{m} g_{k}\left(\delta_{1}, \delta_{2}, \delta_{3}\right)\right| \diamond \delta_{1} \diamond \delta_{2} \diamond \delta_{3} \\
& \quad \leq \prod_{k=1}^{s}\left(\int_{\xi_{1}}^{\sigma_{1}} \int_{\xi_{2}}^{\sigma_{2}} \int_{\xi_{3}}^{\sigma_{3}}\left|h\left(\delta_{1}, \delta_{2}, \delta_{3}\right)\right|\left|g_{k}\left(\delta_{1}, \delta_{2}, \delta_{3}\right)\right|^{p_{k}} \diamond \delta_{1} \diamond \delta_{2} \diamond \delta_{3}\right)^{1 / p_{k}} .
\end{aligned}
$$

Thus, we have

$$
\begin{aligned}
& \int_{\xi_{1}}^{\sigma_{1}} \int_{\xi_{2}}^{\sigma_{2}} \int_{\xi_{3}}^{\sigma_{3}}\left|h\left(\delta_{1}, \delta_{2}, \delta_{3}\right)\right|\left|\prod_{j=1}^{m} f_{j}\left(\delta_{1}, \delta_{2}, \delta_{3}\right)\right| \diamond \delta_{1} \diamond \delta_{2} \diamond \delta_{3} \\
& \leq \prod_{k=1}^{s}\left(\int_{\xi_{1}}^{\sigma_{1}} \int_{\xi_{2}}^{\sigma_{2}} \int_{\xi_{3}}^{\sigma_{3}}\left|h\left(\delta_{1}, \delta_{2}, \delta_{3}\right)\right| \prod_{j=1}^{m}\left|f_{j}\left(\delta_{1}, \delta_{2}, \delta_{3}\right)\right|^{1+p_{k} \alpha_{k j}} \diamond \delta_{1} \diamond \delta_{2} \diamond \delta_{3}\right)^{1 / p_{k}}
\end{aligned}
$$

(2) The proof of inequality (23) is similar to the proof of inequality (22), we have

$$
\begin{aligned}
& \int_{\xi_{1}}^{\sigma_{1}} \int_{\xi_{2}}^{\sigma_{2}} \int_{\xi_{3}}^{\sigma_{3}}\left|h\left(\delta_{1}, \delta_{2}, \delta_{3}\right)\right|\left|\prod_{j=1}^{m} g_{k}\left(\delta_{1}, \delta_{2}, \delta_{3}\right)\right| \diamond \delta_{1} \diamond \delta_{2} \diamond \delta_{3} \\
& \geq \prod_{k=1}^{s}\left(\int_{\xi_{1}}^{\sigma_{1}} \int_{\xi_{2}}^{\sigma_{2}} \int_{\xi_{3}}^{\sigma_{3}}\left|h\left(\delta_{1}, \delta_{2}, \delta_{3}\right)\right|\left|g_{k}\left(\delta_{1}, \delta_{2}, \delta_{3}\right)\right|^{p_{k}} \diamond \delta_{1} \diamond \delta_{2} \diamond \delta_{3}\right)^{1 / p_{k}} .
\end{aligned}
$$


Thus, we have

$$
\begin{aligned}
& \int_{\xi_{1}}^{\sigma_{1}} \int_{\xi_{2}}^{\sigma_{2}} \int_{\xi_{3}}^{\sigma_{3}}\left|h\left(\delta_{1}, \delta_{2}, \delta_{3}\right)\right|\left|\prod_{j=1}^{m} f_{j}\left(\delta_{1}, \delta_{2}, \delta_{3}\right)\right| \diamond \delta_{1} \diamond \delta_{2} \diamond \delta_{3} \\
& \geq \prod_{k=1}^{s}\left(\int_{\xi_{1}}^{\sigma_{1}} \int_{\xi_{2}}^{\sigma_{2}} \int_{\xi_{3}}^{\sigma_{3}}\left|h\left(\delta_{1}, \delta_{2}, \delta_{3}\right)\right| \prod_{j=1}^{m}\left|f_{j}\left(\delta_{1}, \delta_{2}, \delta_{3}\right)\right|^{1+p_{k} \alpha_{k j}} \diamond \delta_{1} \diamond \delta_{2} \diamond \delta_{3}\right)^{1 / p_{k}}
\end{aligned}
$$

Thus, the proof of Theorem 3.8 is completed.

Corollary 3.9 Under the assumptions of Theorem 3.8, taking $s=m, \alpha_{k j}=-t / p_{k}$ for $j \neq k$ and $\alpha_{k k}=t\left(1-1 / p_{k}\right)$ with $t \in \mathbb{R}$, the following assertions hold true.

(1) For $p_{k}>1$, one has

$$
\begin{aligned}
& \int_{\xi_{1}}^{\sigma_{1}} \int_{\xi_{2}}^{\sigma_{2}} \int_{\xi_{3}}^{\sigma_{3}}\left|h\left(\delta_{1}, \delta_{2}, \delta_{3}\right)\right|\left|\prod_{j=1}^{m} f_{j}\left(\delta_{1}, \delta_{2}, \delta_{3}\right)\right| \diamond \delta_{1} \diamond \delta_{2} \diamond \delta_{3} \\
& \leq \prod_{k=1}^{m}\left(\int_{\xi_{1}}^{\sigma_{1}} \int_{\xi_{2}}^{\sigma_{2}} \int_{\xi_{3}}^{\sigma_{3}}\left|h\left(\delta_{1}, \delta_{2}, \delta_{3}\right)\right|\left(\prod_{j=1}^{m}\left|f_{j}\left(\delta_{1}, \delta_{2}, \delta_{3}\right)\right|\right)^{1-t}\right. \\
& \left.\quad \times\left(\left|f_{k}\left(\delta_{1}, \delta_{2}, \delta_{3}\right)\right|^{p_{k}}\right)^{t} \diamond \delta_{1} \diamond \delta_{2} \diamond \delta_{3}\right)^{1 / p_{k}}
\end{aligned}
$$

(2) For $0<p_{m}<1, p_{k}<0(k=1,2, \ldots, m-1)$, one has

$$
\begin{aligned}
\int_{\xi_{1}}^{\sigma_{1}} \int_{\xi_{2}}^{\sigma_{2}} \int_{\xi_{3}}^{\sigma_{3}}\left|h\left(\delta_{1}, \delta_{2}, \delta_{3}\right)\right|\left|\prod_{j=1}^{m} f_{j}\left(\delta_{1}, \delta_{2}, \delta_{3}\right)\right| \diamond \delta_{1} \diamond \delta_{2} \diamond \delta_{3} \\
\geq \prod_{k=1}^{m}\left(\int_{\xi_{1}}^{\sigma_{1}} \int_{\xi_{2}}^{\sigma_{2}} \int_{\xi_{3}}^{\sigma_{3}}\left|h\left(\delta_{1}, \delta_{2}, \delta_{3}\right)\right|\left(\prod_{j=1}^{m}\left|f_{j}\left(\delta_{1}, \delta_{2}, \delta_{3}\right)\right|\right)^{1-t}\right. \\
\left.\quad \times\left(\left|f_{k}\left(\delta_{1}, \delta_{2}, \delta_{3}\right)\right|^{p_{k}}\right)^{t} \diamond \delta_{1} \diamond \delta_{2} \diamond \delta_{3}\right)^{1 / p_{k}} .
\end{aligned}
$$

Theorem 3.10 Assume that $\mathbb{T}$ is a time scale, $\xi, \sigma \in \mathbb{T}$ with $\xi<\sigma$ and $p_{k}>0, \alpha_{k j} \in \mathbb{R}(j=$ $1,2, \ldots, m, k=1,2, \ldots, s), \sum_{k=1}^{s} \frac{1}{p_{k}}=1, \sum_{k=1}^{s} \alpha_{k j}=0, f_{j}, h: \mathbb{T} \rightarrow \mathbb{R}$. If $h$ and $f_{j}$ are $\diamond$-integrable on $[\xi, \sigma]_{\mathbb{T}}$, then the following assertions hold true.

(1) For $p_{k}>1$, one has

$$
\begin{aligned}
\int_{\xi_{1}}^{\sigma_{1}} & \int_{\xi_{2}}^{\sigma_{2}} \cdots \int_{\xi_{n}}^{\sigma_{n}}\left|h\left(\delta_{1}, \delta_{2}, \ldots, \delta_{n}\right)\right|\left|\prod_{j=1}^{m} f_{j}\left(\delta_{1}, \delta_{2}, \ldots, \delta_{n}\right)\right| \nabla \delta_{1} \diamond \delta_{2} \cdots \diamond \delta_{n} \\
\leq & \prod_{k=1}^{s}\left(\int_{\xi_{1}}^{\sigma_{1}} \int_{\xi_{2}}^{\sigma_{2}} \cdots \int_{\xi_{n}}^{\sigma_{n}}\left|h\left(\delta_{1}, \delta_{2}, \ldots, \delta_{n}\right)\right|\right. \\
& \left.\times \prod_{j=1}^{m}\left|f_{j}\left(\delta_{1}, \delta_{2}, \ldots, \delta_{n}\right)\right|^{1+p_{k} \alpha_{k j}} \diamond \delta_{1} \diamond \delta_{2} \cdots \diamond \delta_{n}\right)^{1 / p_{k}}
\end{aligned}
$$


(2) For $0<p_{s}<1, p_{k}<0(k=1,2, \ldots, s-1), f_{j}^{1+p_{k} \alpha_{k j}}$ is $\diamond$-integrable on $[\xi, \sigma]_{\mathbb{T}}$, one has

$$
\begin{aligned}
\int_{\xi_{1}}^{\sigma_{1}} \int_{\xi_{2}}^{\sigma_{2}} \cdots \int_{\xi_{n}}^{\sigma_{n}}\left|h\left(\delta_{1}, \delta_{2}, \ldots, \delta_{n}\right)\right|\left|\prod_{j=1}^{m} f_{j}\left(\delta_{1}, \delta_{2}, \ldots, \delta_{n}\right)\right| \nabla \delta_{1} \diamond \delta_{2} \cdots \diamond \delta_{n} \\
\geq \prod_{k=1}^{s}\left(\int_{\xi_{1}}^{\sigma_{1}} \int_{\xi_{2}}^{\sigma_{2}} \cdots \int_{\xi_{n}}^{\sigma_{n}}\left|h\left(\delta_{1}, \delta_{2}, \ldots, \delta_{n}\right)\right|\right. \\
\left.\quad \times \prod_{j=1}^{m}\left|f_{j}\left(\delta_{1}, \delta_{2}, \ldots, \delta_{n}\right)\right|^{1+p_{k} \alpha_{k j}} \diamond \delta_{1} \diamond \delta_{2} \cdots \diamond \delta_{n}\right)^{1 / p_{k}}
\end{aligned}
$$

Proof Similar to the proof of Theorem 3.8, we get the result of Theorem 3.10.

Remark 3.11 The inequalities in Theorem 3.8 and Theorem 3.10 are the result of generalization of Theorem 4.1 in Ref. [17].

Theorem 3.12 Assume that $\mathbb{T}$ is a time scale, $\xi, \sigma \in \mathbb{T}$ with $\xi<\sigma$ and $p_{k}>0, r \in \mathbb{R}, \alpha_{k j} \in$ $\mathbb{R}(j=1,2, \ldots, m, k=1,2, \ldots, s), \sum_{k=1}^{s} \frac{1}{p_{k}}=r, \sum_{k=1}^{s} \alpha_{k j}=0, f_{j}, h: \mathbb{T} \rightarrow \mathbb{R}$. If $f_{j}$ and $h$ are $\diamond-$ integrable on $[\xi, \sigma]_{T}$, then the following assertions hold true.

(1) For rp $_{k}>1$, one has

$$
\begin{aligned}
& \int_{\xi_{1}}^{\sigma_{1}} \int_{\xi_{2}}^{\sigma_{2}} \int_{\xi_{3}}^{\sigma_{3}}\left|h\left(\delta_{1}, \delta_{2}, \delta_{3}\right)\right|\left|\prod_{j=1}^{m} f_{j}\left(\delta_{1}, \delta_{2}, \delta_{3}\right)\right| \diamond \delta_{1} \diamond \delta_{2} \diamond \delta_{3} \\
& \quad \leq \prod_{k=1}^{s}\left(\int_{\xi_{1}}^{\sigma_{1}} \int_{\xi_{2}}^{\sigma_{2}} \int_{\xi_{3}}^{\sigma_{3}}\left|h\left(\delta_{1}, \delta_{2}, \delta_{3}\right)\right| \prod_{j=1}^{m}\left|f_{j}\left(\delta_{1}, \delta_{2}, \delta_{3}\right)\right|^{1+r p_{k} \alpha_{k j}} \diamond \delta_{1} \diamond \delta_{2} \diamond \delta_{3}\right)^{1 / r p_{k}} .
\end{aligned}
$$

(2) For $0<r p_{k}<1, r p_{k}<0(k=1,2, \ldots, s-1), f_{j}^{1+r p_{k} \alpha_{k j}}$ is $\diamond$-integrable on $[\xi, \sigma]_{\mathbb{T}}$, one has

$$
\begin{aligned}
& \int_{\xi_{1}}^{\sigma_{1}} \int_{\xi_{2}}^{\sigma_{2}} \int_{\xi_{3}}^{\sigma_{3}}\left|h\left(\delta_{1}, \delta_{2}, \delta_{3}\right)\right|\left|\prod_{j=1}^{m} f_{j}\left(\delta_{1}, \delta_{2}, \delta_{3}\right)\right| \diamond \delta_{1} \diamond \delta_{2} \diamond \delta_{3} \\
& \geq \prod_{k=1}^{s}\left(\int_{\xi_{1}}^{\sigma_{1}} \int_{\xi_{2}}^{\sigma_{2}} \int_{\xi_{3}}^{\sigma_{3}}\left|h\left(\delta_{1}, \delta_{2}, \delta_{3}\right)\right| \prod_{j=1}^{m}\left|f_{j}\left(\delta_{1}, \delta_{2}, \delta_{3}\right)\right|^{1+r p_{k} \alpha_{k j}} \diamond \delta_{1} \diamond \delta_{2} \diamond \delta_{3}\right)^{1 / r p_{k}} .
\end{aligned}
$$

Proof (1) Since $r p_{k}>1$ and $\sum_{k=1}^{s} \frac{1}{r p_{k}}=1$. Then by inequality (22) we can obtain inequality (26).

(2) Since $0<r p_{s}<1, r p_{k}<0$ and $\sum_{k=1}^{s} \frac{1}{r p_{k}}=1$, by inequality (23), we can obtain inequality (27).

The proof of Theorem 3.12 is completed.

Theorem 3.13 Assume that $\mathbb{T}$ is a time scale, $\xi, \sigma \in \mathbb{T}$ with $\xi<\sigma$ and $p_{k}>0, r \in \mathbb{R}, \alpha_{k j} \in$ $\mathbb{R}(j=1,2, \ldots, m, k=1,2, \ldots, s), \sum_{k=1}^{s} \frac{1}{p_{k}}=r, \sum_{k=1}^{s} \alpha_{k j}=0, f_{j}, h: \mathbb{T} \rightarrow \mathbb{R}$. If $f_{j}$ and $h$ are $\diamond-$ integrable on $[\xi, \sigma]_{T}$, then the following assertions hold true. 
(1) For rp $_{k}>1$, one has

$$
\begin{aligned}
\int_{\xi_{1}}^{\sigma_{1}} & \int_{\xi_{2}}^{\sigma_{2}} \cdots \int_{\xi_{n}}^{\sigma_{n}}\left|h\left(\delta_{1}, \delta_{2}, \ldots, \delta_{n}\right)\right|\left|\prod_{j=1}^{m} f_{j}\left(\delta_{1}, \delta_{2}, \ldots, \delta_{n}\right)\right| \nabla \delta_{1} \diamond \delta_{2} \cdots \diamond \delta_{n} \\
\leq & \prod_{k=1}^{s}\left(\int_{\xi_{1}}^{\sigma_{1}} \int_{\xi_{2}}^{\sigma_{2}} \cdots \int_{\xi_{n}}^{\sigma_{n}}\left|h\left(\delta_{1}, \delta_{2}, \ldots, \delta_{n}\right)\right|\right. \\
& \left.\times \prod_{j=1}^{m}\left|f_{j}\left(\delta_{1}, \delta_{2}, \ldots, \delta_{n}\right)\right|^{1+r p_{k} \alpha_{k j}} \diamond \delta_{1} \diamond \delta_{2} \cdots \diamond \delta_{n}\right)^{1 / r p_{k}} .
\end{aligned}
$$

(2) For $0<r p_{k}<1, r p_{k}<0(k=1,2, \ldots, s-1), f_{j}^{1+r p_{k} \alpha_{k j}}$ is $\diamond$-integrable on $[\xi, \sigma]_{\mathbb{T}}$, one has

$$
\begin{aligned}
\int_{\xi_{1}}^{\sigma_{1}} \int_{\xi_{2}}^{\sigma_{2}} \cdots \int_{\xi_{n}}^{\sigma_{n}}\left|h\left(\delta_{1}, \delta_{2}, \ldots, \delta_{n}\right)\right|\left|\prod_{j=1}^{m} f_{j}\left(\delta_{1}, \delta_{2}, \ldots, \delta_{n}\right)\right| \nabla \delta_{1} \diamond \delta_{2} \ldots \diamond \delta_{n} \\
\geq \prod_{k=1}^{s}\left(\int_{\xi_{1}}^{\sigma_{1}} \int_{\xi_{2}}^{\sigma_{2}} \cdots \int_{\xi_{n}}^{\sigma_{n}}\left|h\left(\delta_{1}, \delta_{2}, \ldots, \delta_{n}\right)\right|\right. \\
\left.\quad \times \prod_{j=1}^{m}\left|f_{j}\left(\delta_{1}, \delta_{2}, \ldots, \delta_{n}\right)\right|^{1+r p_{k} \alpha_{k j}} \diamond \delta_{1} \diamond \delta_{2} \cdots \diamond \delta_{n}\right)^{1 / r p_{k}} .
\end{aligned}
$$

Proof Similar to the proof of Theorem 3.12, we get the result of Theorem 3.13.

Theorem 3.14 Let $f, g, h: \mathbb{T} \rightarrow \mathbb{R}$ be $\diamond$-integrable on $[\xi, \sigma]_{T}$, and $s, t \in \mathbb{R}$, and let $p=(s-$ $t) /(1-t), q=(s-t) /(s-1)$.

(1) If $s<1<t$ or $s>1>t$, then

$$
\begin{aligned}
\int_{\xi_{1}}^{\sigma_{1}} & \int_{\xi_{2}}^{\sigma_{2}} \int_{\xi_{3}}^{\sigma_{3}}\left|h\left(\delta_{1}, \delta_{2}, \delta_{3}\right)\right|\left|f\left(\delta_{1}, \delta_{2}, \delta_{3}\right) g\left(\delta_{1}, \delta_{2}, \delta_{3}\right)\right| \diamond \delta_{1} \diamond \delta_{2} \diamond \delta_{3} \\
\leq & \left(\int_{\xi_{1}}^{\sigma_{1}} \int_{\xi_{2}}^{\sigma_{2}} \int_{\xi_{3}}^{\sigma_{3}}\left|h\left(\delta_{1}, \delta_{2}, \delta_{3}\right)\right|\left|f\left(\delta_{1}, \delta_{2}, \delta_{3}\right)\right|^{s p} \diamond \delta_{1} \diamond \delta_{2} \diamond \delta_{3}\right)^{1 / p^{2}} \\
& \times\left(\int_{\xi_{1}}^{\sigma_{1}} \int_{\xi_{2}}^{\sigma_{2}} \int_{\xi_{3}}^{\sigma_{3}}\left|h\left(\delta_{1}, \delta_{2}, \delta_{3}\right)\right|\left|g\left(\delta_{1}, \delta_{2}, \delta_{3}\right)\right|^{t q} \diamond \delta_{1} \diamond \delta_{2} \diamond \delta_{3}\right)^{1 / q^{2}} \\
& \times\left(\int_{\xi_{1}}^{\sigma_{1}} \int_{\xi_{2}}^{\sigma_{2}} \int_{\xi_{3}}^{\sigma_{3}}\left|h\left(\delta_{1}, \delta_{2}, \delta_{3}\right)\right|\left|f\left(\delta_{1}, \delta_{2}, \delta_{3}\right)\right|^{t p} \diamond \delta_{1} \diamond \delta_{2} \diamond \delta_{3}\right. \\
& \left.\times \int_{\xi_{1}}^{\sigma_{1}} \int_{\xi_{2}}^{\sigma_{2}} \int_{\xi_{3}}^{\sigma_{3}}\left|h\left(\delta_{1}, \delta_{2}, \delta_{3}\right)\right|\left|g\left(\delta_{1}, \delta_{2}, \delta_{3}\right)\right|^{s q} \diamond \delta_{1} \diamond \delta_{2} \diamond \delta_{3}\right)^{1 / p q} .
\end{aligned}
$$

(2) If $s>t>1$ or $s<t<1$; $>s>1$ or $t<s<1$, then

$$
\begin{gathered}
\int_{\xi_{1}}^{\sigma_{1}} \int_{\xi_{2}}^{\sigma_{2}} \int_{\xi_{3}}^{\sigma_{3}}\left|h\left(\delta_{1}, \delta_{2}, \delta_{3}\right)\right|\left|f\left(\delta_{1}, \delta_{2}, \delta_{3}\right) g\left(\delta_{1}, \delta_{2}, \delta_{3}\right)\right| \diamond \delta_{1} \diamond \delta_{2} \diamond \delta_{3} \\
\geq\left(\int_{\xi_{1}}^{\sigma_{1}} \int_{\xi_{2}}^{\sigma_{2}} \int_{\xi_{3}}^{\sigma_{3}}\left|h\left(\delta_{1}, \delta_{2}, \delta_{3}\right)\right|\left|f\left(\delta_{1}, \delta_{2}, \delta_{3}\right)\right|^{s p} \diamond \delta_{1} \diamond \delta_{2} \diamond \delta_{3}\right)^{1 / p^{2}}
\end{gathered}
$$




$$
\begin{aligned}
& \times\left(\int_{\xi_{1}}^{\sigma_{1}} \int_{\xi_{2}}^{\sigma_{2}} \int_{\xi_{3}}^{\sigma_{3}}\left|h\left(\delta_{1}, \delta_{2}, \delta_{3}\right)\right|\left|g\left(\delta_{1}, \delta_{2}, \delta_{3}\right)\right|^{t q} \diamond \delta_{1} \diamond \delta_{2} \diamond \delta_{3}\right)^{1 / q^{2}} \\
& \times\left(\int_{\xi_{1}}^{\sigma_{1}} \int_{\xi_{2}}^{\sigma_{2}} \int_{\xi_{3}}^{\sigma_{3}}\left|h\left(\delta_{1}, \delta_{2}, \delta_{3}\right)\right|\left|f\left(\delta_{1}, \delta_{2}, \delta_{3}\right)\right|^{t p} \diamond \delta_{1} \diamond \delta_{2} \diamond \delta_{3}\right. \\
& \left.\times \int_{\xi_{1}}^{\sigma_{1}} \int_{\xi_{2}}^{\sigma_{2}} \int_{\xi_{3}}^{\sigma_{3}}\left|h\left(\delta_{1}, \delta_{2}, \delta_{3}\right)\right|\left|g\left(\delta_{1}, \delta_{2}, \delta_{3}\right)\right|^{s q} \diamond \delta_{1} \diamond \delta_{2} \diamond \delta_{3}\right)^{1 / p q} .
\end{aligned}
$$

Proof (1) Let $p=\frac{s-t}{1-t}$ and in view of $s<1<t$ or $s>1>t$, we have

$$
p=\frac{s-t}{1-t}>1
$$

by Hölder's inequality (5) with indices $\frac{s-t}{1-t}$ and $\frac{s-t}{s-1}$, we have

$$
\begin{aligned}
\int_{\xi_{1}}^{\sigma_{1}} & \int_{\xi_{2}}^{\sigma_{2}} \int_{\xi_{3}}^{\sigma_{3}}|h||f g| \diamond \delta_{1} \diamond \delta_{2} \diamond \delta_{3} \\
= & \int_{\xi_{1}}^{\sigma_{1}} \int_{\xi_{2}}^{\sigma_{2}} \int_{\xi_{3}}^{\sigma_{3}}|h||f g|^{s(1-t) /(s-t)}|f g|^{t(s-1) /(s-t)} \diamond \delta_{1} \diamond \delta_{2} \diamond \delta_{3} \\
\leq & \left(\int_{\xi_{1}}^{\sigma_{1}} \int_{\xi_{2}}^{\sigma_{2}} \int_{\xi_{3}}^{\sigma_{3}}|h||f g|^{s} \diamond \delta_{1} \diamond \delta_{2} \diamond \delta_{3}\right)^{(1-t) /(s-t)} \\
& \times\left(\int_{\xi_{1}}^{\sigma_{1}} \int_{\xi_{2}}^{\sigma_{2}} \int_{\xi_{3}}^{\sigma_{3}}|h||f g|^{t} \diamond \delta_{1} \diamond \delta_{2} \diamond \delta_{3}\right)^{(s-1) /(s-t)} .
\end{aligned}
$$

On the other hand, from Hölder's inequality (5) again for $p=\frac{s-t}{1-t}>1$, it follows that the following two inequalities are true:

$$
\begin{aligned}
\int_{\xi_{1}}^{\sigma_{1}} & \int_{\xi_{2}}^{\sigma_{2}} \int_{\xi_{3}}^{\sigma_{3}}|h||f g|^{s} \diamond \delta_{1} \diamond \delta_{2} \diamond \delta_{3} \\
\leq & \left(\int_{\xi_{1}}^{\sigma_{1}} \int_{\xi_{2}}^{\sigma_{2}} \int_{\xi_{3}}^{\sigma_{3}}|h||f|^{s(s-t) /(1-t)} \diamond \delta_{1} \diamond \delta_{2} \diamond \delta_{3}\right)^{(1-t) /(s-t)} \\
& \times\left(\int_{\xi_{1}}^{\sigma_{1}} \int_{\xi_{2}}^{\sigma_{2}} \int_{\xi_{3}}^{\sigma_{3}}|h||g|^{s(s-t) /(s-1)} \diamond \delta_{1} \diamond \delta_{2} \diamond \delta_{3}\right)^{(s-1) /(s-t)}
\end{aligned}
$$

and

$$
\begin{aligned}
\int_{\xi_{1}}^{\sigma_{1}} & \int_{\xi_{2}}^{\sigma_{2}} \int_{\xi_{3}}^{\sigma_{3}}|h||f g|^{t} \diamond \delta_{1} \diamond \delta_{2} \diamond \delta_{3} \\
\leq & \left(\int_{\xi_{1}}^{\sigma_{1}} \int_{\xi_{2}}^{\sigma_{2}} \int_{\xi_{3}}^{\sigma_{3}}|h||f|^{t(s-t) /(1-t)} \diamond \delta_{1} \diamond \delta_{2} \diamond \delta_{3}\right)^{(1-t) /(s-t)} \\
& \times\left(\int_{\xi_{1}}^{\sigma_{1}} \int_{\xi_{2}}^{\sigma_{2}} \int_{\xi_{3}}^{\sigma_{3}}|h||g|^{t(s-t) /(s-1)} \diamond \delta_{1} \diamond \delta_{2} \diamond \delta_{3}\right)^{(s-1) /(s-t)} .
\end{aligned}
$$

Thus, we have

$$
\int_{\xi_{1}}^{\sigma_{1}} \int_{\xi_{2}}^{\sigma_{2}} \int_{\xi_{3}}^{\sigma_{3}}\left|h\left(\delta_{1}, \delta_{2}, \delta_{3}\right)\right|\left|f\left(\delta_{1}, \delta_{2}, \delta_{3}\right) g\left(\delta_{1}, \delta_{2}, \delta_{3}\right)\right| \diamond \delta_{1} \diamond \delta_{2} \diamond \delta_{3}
$$




$$
\begin{aligned}
\leq & \left(\int_{\xi_{1}}^{\sigma_{1}} \int_{\xi_{2}}^{\sigma_{2}} \int_{\xi_{3}}^{\sigma_{3}}\left|h\left(\delta_{1}, \delta_{2}, \delta_{3}\right)\right|\left|f\left(\delta_{1}, \delta_{2}, \delta_{3}\right)\right|^{s p} \diamond \delta_{1} \diamond \delta_{2} \diamond \delta_{3}\right)^{1 / p^{2}} \\
& \times\left(\int_{\xi_{1}}^{\sigma_{1}} \int_{\xi_{2}}^{\sigma_{2}} \int_{\xi_{3}}^{\sigma_{3}}\left|h\left(\delta_{1}, \delta_{2}, \delta_{3}\right)\right|\left|g\left(\delta_{1}, \delta_{2}, \delta_{3}\right)\right|^{t q} \diamond \delta_{1} \diamond \delta_{2} \diamond \delta_{3}\right)^{1 / q^{2}} \\
& \times\left(\int_{\xi_{1}}^{\sigma_{1}} \int_{\xi_{2}}^{\sigma_{2}} \int_{\xi_{3}}^{\sigma_{3}}\left|h\left(\delta_{1}, \delta_{2}, \delta_{3}\right)\right|\left|f\left(\delta_{1}, \delta_{2}, \delta_{3}\right)\right|^{t p} \diamond \delta_{1} \diamond \delta_{2} \diamond \delta_{3}\right. \\
& \left.\times \int_{\xi_{1}}^{\sigma_{1}} \int_{\xi_{2}}^{\sigma_{2}} \int_{\xi_{3}}^{\sigma_{3}}\left|h\left(\delta_{1}, \delta_{2}, \delta_{3}\right)\right|\left|g\left(\delta_{1}, \delta_{2}, \delta_{3}\right)\right|^{s q} \diamond \delta_{1} \diamond \delta_{2} \diamond \delta_{3}\right)^{1 / p q} .
\end{aligned}
$$

(2) Let $p=\frac{s-t}{1-t}$ and in view of $s>t>1$ or $s<t<1$, we have

$$
p=\frac{s-t}{1-t}<0
$$

and $t>s>1$ or $t<s<1$, we have $0<\frac{s-t}{1-t}<1$, by the reverse Hölder inequality (6) with indices $\frac{s-t}{1-t}$ and $\frac{s-t}{s-1}$, we have

$$
\begin{aligned}
\int_{\xi_{1}}^{\sigma_{1}} & \int_{\xi_{2}}^{\sigma_{2}} \int_{\xi_{3}}^{\sigma_{3}}|h||f g| \diamond \delta_{1} \diamond \delta_{2} \diamond \delta_{3} \\
= & \int_{\xi_{1}}^{\sigma_{1}} \int_{\xi_{2}}^{\sigma_{2}} \int_{\xi_{3}}^{\sigma_{3}}|h||f g|^{s(1-t) /(s-t)}|f g|^{t(s-1) /(s-t)} \diamond \delta_{1} \diamond \delta_{2} \diamond \delta_{3} \\
\geq & \left(\int_{\xi_{1}}^{\sigma_{1}} \int_{\xi_{2}}^{\sigma_{2}} \int_{\xi_{3}}^{\sigma_{3}}|h||f g|^{s} \diamond \delta_{1} \diamond \delta_{2} \diamond \delta_{3}\right)^{(1-t) /(s-t)} \\
& \times\left(\int_{\xi_{1}}^{\sigma_{1}} \int_{\xi_{2}}^{\sigma_{2}} \int_{\xi_{3}}^{\sigma_{3}}|h||f g|^{t} \diamond \delta_{1} \diamond \delta_{2} \diamond \delta_{3}\right)^{(s-1) /(s-t)} .
\end{aligned}
$$

On the other hand, from Hölder's inequality (6) again for $0<p=\frac{s-t}{1-t}<1$ or $p=\frac{s-t}{1-t}<0$, it follows that the following two inequalities are true:

$$
\begin{aligned}
\int_{\xi_{1}}^{\sigma_{1}} & \int_{\xi_{2}}^{\sigma_{2}} \int_{\xi_{3}}^{\sigma_{3}}|h||f g|^{s} \diamond \delta_{1} \diamond \delta_{2} \diamond \delta_{3} \\
\geq & \left(\int_{\xi_{1}}^{\sigma_{1}} \int_{\xi_{2}}^{\sigma_{2}} \int_{\xi_{3}}^{\sigma_{3}}|h||f|^{s(s-t) /(1-t)} \diamond \delta_{1} \diamond \delta_{2} \diamond \delta_{3}\right)^{(1-t) /(s-t)} \\
& \times\left(\int_{\xi_{1}}^{\sigma_{1}} \int_{\xi_{2}}^{\sigma_{2}} \int_{\xi_{3}}^{\sigma_{3}}|h||g|^{s(s-t) /(s-1)} \diamond \delta_{1} \diamond \delta_{2} \diamond \delta_{3}\right)^{(s-1) /(s-t)}
\end{aligned}
$$

and

$$
\begin{aligned}
\int_{\xi_{1}}^{\sigma_{1}} & \int_{\xi_{2}}^{\sigma_{2}} \int_{\xi_{3}}^{\sigma_{3}}|h||f g|^{t} \diamond \delta_{1} \diamond \delta_{2} \diamond \delta_{3} \\
\geq & \left(\int_{\xi_{1}}^{\sigma_{1}} \int_{\xi_{2}}^{\sigma_{2}} \int_{\xi_{3}}^{\sigma_{3}}|h||f|^{t(s-t) /(1-t)} \diamond \delta_{1} \diamond \delta_{2} \diamond \delta_{3}\right)^{(1-t) /(s-t)} \\
& \times\left(\int_{\xi_{1}}^{\sigma_{1}} \int_{\xi_{2}}^{\sigma_{2}} \int_{\xi_{3}}^{\sigma_{3}}|h||g|^{t(s-t) /(s-1)} \diamond \delta_{1} \diamond \delta_{2} \diamond \delta_{3}\right)^{(s-1) /(s-t)} .
\end{aligned}
$$


Thus, we have

$$
\begin{aligned}
& \int_{\xi_{1}}^{\sigma_{1}} \int_{\xi_{2}}^{\sigma_{2}} \int_{\xi_{3}}^{\sigma_{3}}\left|h\left(\delta_{1}, \delta_{2}, \delta_{3}\right)\right|\left|f\left(\delta_{1}, \delta_{2}, \delta_{3}\right) g\left(\delta_{1}, \delta_{2}, \delta_{3}\right)\right| \diamond \delta_{1} \diamond \delta_{2} \diamond \delta_{3} \\
& \geq\left(\int_{\xi_{1}}^{\sigma_{1}} \int_{\xi_{2}}^{\sigma_{2}} \int_{\xi_{3}}^{\sigma_{3}}\left|h\left(\delta_{1}, \delta_{2}, \delta_{3}\right)\right|\left|f\left(\delta_{1}, \delta_{2}, \delta_{3}\right)\right|^{s p} \diamond \delta_{1} \diamond \delta_{2} \diamond \delta_{3}\right)^{1 / p^{2}} \\
& \times\left(\int_{\xi_{1}}^{\sigma_{1}} \int_{\xi_{2}}^{\sigma_{2}} \int_{\xi_{3}}^{\sigma_{3}}\left|h\left(\delta_{1}, \delta_{2}, \delta_{3}\right)\right|\left|g\left(\delta_{1}, \delta_{2}, \delta_{3}\right)\right|^{t q} \diamond \delta_{1} \diamond \delta_{2} \diamond \delta_{3}\right)^{1 / q^{2}} \\
& \times\left(\int_{\xi_{1}}^{\sigma_{1}} \int_{\xi_{2}}^{\sigma_{2}} \int_{\xi_{3}}^{\sigma_{3}}\left|h\left(\delta_{1}, \delta_{2}, \delta_{3}\right)\right|\left|f\left(\delta_{1}, \delta_{2}, \delta_{3}\right)\right|^{t p} \diamond \delta_{1} \diamond \delta_{2} \diamond \delta_{3}\right. \\
&\left.\times \int_{\xi_{1}}^{\sigma_{1}} \int_{\xi_{2}}^{\sigma_{2}} \int_{\xi_{3}}^{\sigma_{3}}\left|h\left(\delta_{1}, \delta_{2}, \delta_{3}\right)\right|\left|g\left(\delta_{1}, \delta_{2}, \delta_{3}\right)\right|^{s q} \diamond \delta_{1} \diamond \delta_{2} \diamond \delta_{3}\right)^{1 / p q} .
\end{aligned}
$$

Thus, the proof of Theorem 3.14 is completed.

Theorem 3.15 Let $f, g, h: \mathbb{T} \rightarrow \mathbb{R}$ be $\diamond$-integrable on $[\xi, \sigma]_{T}$, and $s, t \in \mathbb{R}$, and let $p=(s-$ $t) /(1-t), q=(s-t) /(s-1)$.

(1) If $s<1<$ or $s>1>t$, then

$$
\begin{aligned}
& \int_{\xi_{1}}^{\sigma_{1}} \int_{\xi_{2}}^{\sigma_{2}} \cdots \int_{\xi_{n}}^{\sigma_{n}}\left|h\left(\delta_{1}, \delta_{2}, \ldots, \delta_{n}\right)\right|\left|f\left(\delta_{1}, \delta_{2}, \ldots, \delta_{n}\right) g\left(\delta_{1}, \delta_{2}, \ldots, \delta_{n}\right)\right| \diamond \delta_{1} \diamond \delta_{2} \ldots \diamond \delta_{n} \\
& \leq\left(\int_{\xi_{1}}^{\sigma_{1}} \int_{\xi_{2}}^{\sigma_{2}} \cdots \int_{\xi_{n}}^{\sigma_{n}}\left|h\left(\delta_{1}, \delta_{2}, \ldots, \delta_{n}\right)\right|\left|f\left(\delta_{1}, \delta_{2}, \ldots, \delta_{n}\right)\right|^{s p} \diamond \delta_{1} \diamond \delta_{2} \cdots \diamond \delta_{n}\right)^{1 / p^{2}} \\
& \times\left(\int_{\xi_{1}}^{\sigma_{1}} \int_{\xi_{2}}^{\sigma_{2}} \cdots \int_{\xi_{n}}^{\sigma_{n}}\left|h\left(\delta_{1}, \delta_{2}, \ldots, \delta_{n}\right)\right|\left|g\left(\delta_{1}, \delta_{2}, \ldots, \delta_{n}\right)\right|^{t q} \diamond \delta_{1} \diamond \delta_{2} \cdots \diamond \delta_{n}\right)^{1 / q^{2}} \\
& \times\left(\int_{\xi_{1}}^{\sigma_{1}} \int_{\xi_{2}}^{\sigma_{2}} \cdots \int_{\xi_{n}}^{\sigma_{n}}\left|h\left(\delta_{1}, \delta_{2}, \ldots, \delta_{n}\right)\right|\left|f\left(\delta_{1}, \delta_{2}, \ldots, \delta_{n}\right)\right|^{t p} \diamond \delta_{1} \diamond \delta_{2} \cdots \diamond \delta_{n}\right. \\
& \left.\times \int_{\xi_{1}}^{\sigma_{1}} \int_{\xi_{2}}^{\sigma_{2}} \cdots \int_{\xi_{n}}^{\sigma_{n}}\left|h\left(\delta_{1}, \delta_{2}, \ldots, \delta_{n}\right)\right|\left|g\left(\delta_{1}, \delta_{2}, \ldots, \delta_{n}\right)\right|^{s q} \diamond \delta_{1} \diamond \delta_{2} \ldots \diamond \delta_{n}\right)^{1 / p q} .
\end{aligned}
$$

(2) If $s>t>1$ or $s<t<1 ; t>s>1$ or $t<s<1$, then

$$
\begin{aligned}
& \int_{\xi_{1}}^{\sigma_{1}} \int_{\xi_{2}}^{\sigma_{2}} \cdots \int_{\xi_{n}}^{\sigma_{n}}\left|h\left(\delta_{1}, \delta_{2}, \ldots, \delta_{n}\right)\right|\left|f\left(\delta_{1}, \delta_{2}, \ldots, \delta_{n}\right) g\left(\delta_{1}, \delta_{2}, \ldots, \delta_{n}\right)\right| \diamond \delta_{1} \diamond \delta_{2} \cdots \diamond \delta_{n} \\
& \geq\left(\int_{\xi_{1}}^{\sigma_{1}} \int_{\xi_{2}}^{\sigma_{2}} \ldots \int_{\xi_{n}}^{\sigma_{n}}\left|h\left(\delta_{1}, \delta_{2}, \ldots, \delta_{n}\right)\right|\left|f\left(\delta_{1}, \delta_{2}, \ldots, \delta_{n}\right)\right|^{s p} \diamond \delta_{1} \diamond \delta_{2} \cdots \diamond \delta_{n}\right)^{1 / p^{2}} \\
& \times\left(\int_{\xi_{1}}^{\sigma_{1}} \int_{\xi_{2}}^{\sigma_{2}} \cdots \int_{\xi_{n}}^{\sigma_{n}}\left|h\left(\delta_{1}, \delta_{2}, \ldots, \delta_{n}\right)\right|\left|g\left(\delta_{1}, \delta_{2}, \ldots, \delta_{n}\right)\right|^{t q} \diamond \delta_{1} \diamond \delta_{2} \cdots \diamond \delta_{n}\right)^{1 / q^{2}} \\
& \times\left(\int_{\xi_{1}}^{\sigma_{1}} \int_{\xi_{2}}^{\sigma_{2}} \cdots \int_{\xi_{n}}^{\sigma_{n}}\left|h\left(\delta_{1}, \delta_{2}, \ldots, \delta_{n}\right)\right|\left|f\left(\delta_{1}, \delta_{2}, \ldots, \delta_{n}\right)\right|^{t p} \diamond \delta_{1} \diamond \delta_{2} \ldots \diamond \delta_{n}\right. \\
& \left.\times \int_{\xi_{1}}^{\sigma_{1}} \int_{\xi_{2}}^{\sigma_{2}} \cdots \int_{\xi_{n}}^{\sigma_{n}}\left|h\left(\delta_{1}, \delta_{2}, \ldots, \delta_{n}\right)\right|\left|g\left(\delta_{1}, \delta_{2}, \ldots, \delta_{n}\right)\right|^{s q} \diamond \delta_{1} \diamond \delta_{2} \ldots \diamond \delta_{n}\right)^{1 / p q} .
\end{aligned}
$$

Proof Similar to the proof of Theorem 3.14, we get the result of Theorem 3.15. 
Remark 3.16 For the inequality of Theorem 5.1 in the Reference [17], we put forward Theorem 3.14 and Theorem 3.15 as the generalization results.

\section{Main results about diamond-alpha integral Minkowski's inequality}

Next, we give some generalizations of diamond- $\alpha$ integral Minkowski's inequality in the following theorems.

Theorem 4.1 Let $f, g, h: \mathbb{T} \rightarrow \mathbb{R}$ be $\diamond$-integrable on $[\xi, \sigma]_{\mathbb{T}}, p>0, s, t \in \mathbb{R}$, and $s \neq t$.

(1) Let $p, s, t \in \mathbb{R}$ be different such that $s, t>1$ and $(s-t) /(p-t)>1$. Then

$$
\begin{aligned}
& \int_{\xi_{1}}^{\sigma_{1}} \int_{\xi_{2}}^{\sigma_{2}} \int_{\xi_{3}}^{\sigma_{3}}\left|h\left(\delta_{1}, \delta_{2}, \delta_{3}\right)\right|\left|f\left(\delta_{1}, \delta_{2}, \delta_{3}\right)+g\left(\delta_{1}, \delta_{2}, \delta_{3}\right)\right|^{p} \diamond \delta_{1} \diamond \delta_{2} \diamond \delta_{3} \\
& \leq {\left[\left(\int_{\xi_{1}}^{\sigma_{1}} \int_{\xi_{2}}^{\sigma_{2}} \int_{\xi_{3}}^{\sigma_{3}}\left|h\left(\delta_{1}, \delta_{2}, \delta_{3}\right)\right|\left|f\left(\delta_{1}, \delta_{2}, \delta_{3}\right)\right|^{s} \diamond \delta_{1} \diamond \delta_{2} \diamond \delta_{3}\right)^{\frac{1}{s}}\right.} \\
&\left.+\left(\int_{\xi_{1}}^{\sigma_{1}} \int_{\xi_{2}}^{\sigma_{2}} \int_{\xi_{3}}^{\sigma_{3}}\left|h\left(\delta_{1}, \delta_{2}, \delta_{3}\right)\right|\left|g\left(\delta_{1}, \delta_{2}, \delta_{3}\right)\right|^{s} \diamond \delta_{1} \diamond \delta_{2} \diamond \delta_{3}\right)^{\frac{1}{s}}\right]^{s(p-t) /(s-t)} \\
& \times\left[\left(\int_{\xi_{1}}^{\sigma_{1}} \int_{\xi_{2}}^{\sigma_{2}} \int_{\xi_{3}}^{\sigma_{3}}\left|h\left(\delta_{1}, \delta_{2}, \delta_{3}\right)\right|\left|f\left(\delta_{1}, \delta_{2}, \delta_{3}\right)\right|^{t} \diamond \delta_{1} \diamond \delta_{2} \diamond \delta_{3}\right)^{\frac{1}{t}}\right. \\
&\left.+\left(\int_{\xi_{1}}^{\sigma_{1}} \int_{\xi_{2}}^{\sigma_{2}} \int_{\xi_{3}}^{\sigma_{3}}\left|h\left(\delta_{1}, \delta_{2}, \delta_{3}\right)\right|\left|g\left(\delta_{1}, \delta_{2}, \delta_{3}\right)\right|^{t} \diamond \delta_{1} \diamond \delta_{2} \diamond \delta_{3}\right)^{\frac{1}{t}}\right]^{t(s-p) /(s-t)} .
\end{aligned}
$$

(2) Let $p, s, t \in \mathbb{R}$ be different such that $0<s<1,0<t<1$ and $(s-t) /(p-t)<1$. Then

$$
\begin{aligned}
& \int_{\xi_{1}}^{\sigma_{1}} \int_{\xi_{2}}^{\sigma_{2}} \int_{\xi_{3}}^{\sigma_{3}}\left|h\left(\delta_{1}, \delta_{2}, \delta_{3}\right)\right|\left|f\left(\delta_{1}, \delta_{2}, \delta_{3}\right)+g\left(\delta_{1}, \delta_{2}, \delta_{3}\right)\right|^{p} \diamond \delta_{1} \diamond \delta_{2} \diamond \delta_{3} \\
& \geq {\left[\left(\int_{\xi_{1}}^{\sigma_{1}} \int_{\xi_{2}}^{\sigma_{2}} \int_{\xi_{3}}^{\sigma_{3}}\left|h\left(\delta_{1}, \delta_{2}, \delta_{3}\right)\right|\left|f\left(\delta_{1}, \delta_{2}, \delta_{3}\right)\right|^{s} \diamond \delta_{1} \diamond \delta_{2} \diamond \delta_{3}\right)^{\frac{1}{s}}\right.} \\
&\left.+\left(\int_{\xi_{1}}^{\sigma_{1}} \int_{\xi_{2}}^{\sigma_{2}} \int_{\xi_{3}}^{\sigma_{3}}\left|h\left(\delta_{1}, \delta_{2}, \delta_{3}\right)\right|\left|g\left(\delta_{1}, \delta_{2}, \delta_{3}\right)\right|^{s} \diamond \delta_{1} \diamond \delta_{2} \diamond \delta_{3}\right)^{\frac{1}{s}}\right]^{s(p-t) /(s-t)} \\
& \times\left[\left(\int_{\xi_{1}}^{\sigma_{1}} \int_{\xi_{2}}^{\sigma_{2}} \int_{\xi_{3}}^{\sigma_{3}}\left|h\left(\delta_{1}, \delta_{2}, \delta_{3}\right)\right|\left|f\left(\delta_{1}, \delta_{2}, \delta_{3}\right)\right|^{t} \diamond \delta_{1} \diamond \delta_{2} \diamond \delta_{3}\right)^{\frac{1}{t}}\right. \\
&\left.+\left(\int_{\xi_{1}}^{\sigma_{1}} \int_{\xi_{2}}^{\sigma_{2}} \int_{\xi_{3}}^{\sigma_{3}}\left|h\left(\delta_{1}, \delta_{2}, \delta_{3}\right)\right|\left|g\left(\delta_{1}, \delta_{2}, \delta_{3}\right)\right|^{t} \diamond \delta_{1} \diamond \delta_{2} \diamond \delta_{3}\right)^{\frac{1}{t}}\right]^{t(s-p) /(s-t)} .
\end{aligned}
$$

Proof (1) We have $(s-t) /(p-t)>1$, and

$$
\begin{aligned}
\int_{\xi_{1}}^{\sigma_{1}} & \int_{\xi_{2}}^{\sigma_{2}} \int_{\xi_{3}}^{\sigma_{3}}\left|h\left(\delta_{1}, \delta_{2}, \delta_{3}\right)\right|\left|f\left(\delta_{1}, \delta_{2}, \delta_{3}\right)+g\left(\delta_{1}, \delta_{2}, \delta_{3}\right)\right|^{p} \diamond \delta_{1} \diamond \delta_{2} \diamond \delta_{3} \\
= & \int_{\xi_{1}}^{\sigma_{1}} \int_{\xi_{2}}^{\sigma_{2}} \int_{\xi_{3}}^{\sigma_{3}}\left|h\left(\delta_{1}, \delta_{2}, \delta_{3}\right)\right|\left(\left|f\left(\delta_{1}, \delta_{2}, \delta_{3}\right)+g\left(\delta_{1}, \delta_{2}, \delta_{3}\right)\right|^{s}\right)^{(p-t) /(s-t)} \\
& \times\left(\left|f\left(\delta_{1}, \delta_{2}, \delta_{3}\right)+g\left(\delta_{1}, \delta_{2}, \delta_{3}\right)\right|^{t}\right)^{(s-p) /(s-t)} \diamond \delta_{1} \diamond \delta_{2} \diamond \delta_{3},
\end{aligned}
$$


by using Hölder's inequality (5) with indices $(s-t) /(p-t)$ and $(s-t) /(s-p)$, we have

$$
\begin{aligned}
\int_{\xi_{1}}^{\sigma_{1}} & \int_{\xi_{2}}^{\sigma_{2}} \int_{\xi_{3}}^{\sigma_{3}}\left|h\left(\delta_{1}, \delta_{2}, \delta_{3}\right)\right|\left|f\left(\delta_{1}, \delta_{2}, \delta_{3}\right)+g\left(\delta_{1}, \delta_{2}, \delta_{3}\right)\right|^{p} \diamond \delta_{1} \diamond \delta_{2} \diamond \delta_{3} \\
\leq & \left(\int_{\xi_{1}}^{\sigma_{1}} \int_{\xi_{2}}^{\sigma_{2}} \int_{\xi_{3}}^{\sigma_{3}}\left|h\left(\delta_{1}, \delta_{2}, \delta_{3}\right)\right|\left|f\left(\delta_{1}, \delta_{2}, \delta_{3}\right)+g\left(\delta_{1}, \delta_{2}, \delta_{3}\right)\right|^{s} \diamond \delta_{1} \diamond \delta_{2} \diamond \delta_{3}\right)^{(p-t) /(s-t)} \\
& \times\left(\int_{\xi_{1}}^{\sigma_{1}} \int_{\xi_{2}}^{\sigma_{2}} \int_{\xi_{3}}^{\sigma_{3}}\left|h\left(\delta_{1}, \delta_{2}, \delta_{3}\right)\right| f\left(\delta_{1}, \delta_{2}, \delta_{3}\right)+\left.g\left(\delta_{1}, \delta_{2}, \delta_{3}\right)\right|^{t} \diamond \delta_{1} \diamond \delta_{2} \diamond \delta_{3}\right)^{(s-p) /(s-t)}
\end{aligned}
$$

On the other hand, by using Minkowski's inequality (6) for $s>1$ and $t>1$, respectively, we can see that the following assertions hold true:

$$
\begin{gathered}
\left(\int_{\xi_{1}}^{\sigma_{1}} \int_{\xi_{2}}^{\sigma_{2}} \int_{\xi_{3}}^{\sigma_{3}}\left|h\left(\delta_{1}, \delta_{2}, \delta_{3}\right)\right|\left|f\left(\delta_{1}, \delta_{2}, \delta_{3}\right)+g\left(\delta_{1}, \delta_{2}, \delta_{3}\right)\right|^{s} \diamond \delta_{1} \diamond \delta_{2} \diamond \delta_{3}\right)^{\frac{1}{s}} \\
\quad \leq\left(\int_{\xi_{1}}^{\sigma_{1}} \int_{\xi_{2}}^{\sigma_{2}} \int_{\xi_{3}}^{\sigma_{3}}\left|h\left(\delta_{1}, \delta_{2}, \delta_{3}\right)\right|\left|f\left(\delta_{1}, \delta_{2}, \delta_{3}\right)\right|^{s} \diamond \delta_{1} \diamond \delta_{2} \diamond \delta_{3}\right)^{\frac{1}{s}} \\
\quad+\left(\int_{\xi_{1}}^{\sigma_{1}} \int_{\xi_{2}}^{\sigma_{2}} \int_{\xi_{3}}^{\sigma_{3}}\left|h\left(\delta_{1}, \delta_{2}, \delta_{3}\right)\right|\left|g\left(\delta_{1}, \delta_{2}, \delta_{3}\right)\right|^{s} \diamond \delta_{1} \diamond \delta_{2} \diamond \delta_{3}\right)^{\frac{1}{s}}
\end{gathered}
$$

and

$$
\begin{gathered}
\left(\int_{\xi_{1}}^{\sigma_{1}} \int_{\xi_{2}}^{\sigma_{2}} \int_{\xi_{3}}^{\sigma_{3}}\left|h\left(\delta_{1}, \delta_{2}, \delta_{3}\right)\right|\left|f\left(\delta_{1}, \delta_{2}, \delta_{3}\right)+g\left(\delta_{1}, \delta_{2}, \delta_{3}\right)\right|^{t} \diamond \delta_{1} \diamond \delta_{2} \diamond \delta_{3}\right)^{\frac{1}{t}} \\
\leq\left(\int_{\xi_{1}}^{\sigma_{1}} \int_{\xi_{2}}^{\sigma_{2}} \int_{\xi_{3}}^{\sigma_{3}}\left|h\left(\delta_{1}, \delta_{2}, \delta_{3}\right)\right|\left|f\left(\delta_{1}, \delta_{2}, \delta_{3}\right)\right|^{t} \diamond \delta_{1} \diamond \delta_{2} \diamond \delta_{3}\right)^{\frac{1}{t}} \\
\quad+\left(\int_{\xi_{1}}^{\sigma_{1}} \int_{\xi_{2}}^{\sigma_{2}} \int_{\xi_{3}}^{\sigma_{3}}\left|h\left(\delta_{1}, \delta_{2}, \delta_{3}\right)\right|\left|g\left(\delta_{1}, \delta_{2}, \delta_{3}\right)\right|^{t} \diamond \delta_{1} \diamond \delta_{2} \diamond \delta_{3}\right)^{\frac{1}{t}} .
\end{gathered}
$$

So we get the result

$$
\begin{aligned}
\int_{\xi_{1}}^{\sigma_{1}} & \int_{\xi_{2}}^{\sigma_{2}} \int_{\xi_{3}}^{\sigma_{3}}\left|h\left(\delta_{1}, \delta_{2}, \delta_{3}\right)\right|\left|f\left(\delta_{1}, \delta_{2}, \delta_{3}\right)+g\left(\delta_{1}, \delta_{2}, \delta_{3}\right)\right|^{p} \diamond \delta_{1} \diamond \delta_{2} \diamond \delta_{3} \\
\leq & {\left[\left(\int_{\xi_{1}}^{\sigma_{1}} \int_{\xi_{2}}^{\sigma_{2}} \int_{\xi_{3}}^{\sigma_{3}}\left|h\left(\delta_{1}, \delta_{2}, \delta_{3}\right)\right|\left|f\left(\delta_{1}, \delta_{2}, \delta_{3}\right)\right|^{s} \diamond \delta_{1} \diamond \delta_{2} \diamond \delta_{3}\right)^{\frac{1}{s}}\right.} \\
& \left.+\left(\int_{\xi_{1}}^{\sigma_{1}} \int_{\xi_{2}}^{\sigma_{2}} \int_{\xi_{3}}^{\sigma_{3}}\left|h\left(\delta_{1}, \delta_{2}, \delta_{3}\right)\right|\left|g\left(\delta_{1}, \delta_{2}, \delta_{3}\right)\right|^{s} \diamond \delta_{1} \diamond \delta_{2} \diamond \delta_{3}\right)^{\frac{1}{s}}\right]^{s(p-t) /(s-t)} \\
& \times\left[\left(\int_{\xi_{1}}^{\sigma_{1}} \int_{\xi_{2}}^{\sigma_{2}} \int_{\xi_{3}}^{\sigma_{3}}\left|h\left(\delta_{1}, \delta_{2}, \delta_{3}\right)\right|\left|f\left(\delta_{1}, \delta_{2}, \delta_{3}\right)\right|^{t} \diamond \delta_{1} \diamond \delta_{2} \diamond \delta_{3}\right)^{\frac{1}{t}}\right. \\
+ & \left.\left(\int_{\xi_{1}}^{\sigma_{1}} \int_{\xi_{2}}^{\sigma_{2}} \int_{\xi_{3}}^{\sigma_{3}}\left|h\left(\delta_{1}, \delta_{2}, \delta_{3}\right)\right|\left|g\left(\delta_{1}, \delta_{2}, \delta_{3}\right)\right|^{t} \diamond \delta_{1} \diamond \delta_{2} \diamond \delta_{3}\right)^{\frac{1}{t}}\right]^{t(s-p) /(s-t)}
\end{aligned}
$$


(2) We have $(s-t) /(p-t)<1$ and

$$
\begin{aligned}
\int_{\xi_{1}}^{\sigma_{1}} & \int_{\xi_{2}}^{\sigma_{2}} \int_{\xi_{3}}^{\sigma_{3}}\left|h\left(\delta_{1}, \delta_{2}, \delta_{3}\right)\right|\left|f\left(\delta_{1}, \delta_{2}, \delta_{3}\right)+g\left(\delta_{1}, \delta_{2}, \delta_{3}\right)\right|^{p} \diamond \delta_{1} \diamond \delta_{2} \diamond \delta_{3} \\
= & \int_{\xi_{1}}^{\sigma_{1}} \int_{\xi_{2}}^{\sigma_{2}} \int_{\xi_{3}}^{\sigma_{3}}\left|h\left(\delta_{1}, \delta_{2}, \delta_{3}\right)\right|\left(\left|f\left(\delta_{1}, \delta_{2}, \delta_{3}\right)+g\left(\delta_{1}, \delta_{2}, \delta_{3}\right)\right|^{s}\right)^{(p-t) /(s-t)} \\
& \times\left(\left|f\left(\delta_{1}, \delta_{2}, \delta_{3}\right)+g\left(\delta_{1}, \delta_{2}, \delta_{3}\right)\right|^{t}\right)^{(s-p) /(s-t)} \diamond \delta_{1} \diamond \delta_{2} \diamond \delta_{3},
\end{aligned}
$$

by using Hölder's inequality (7) with indices $(s-t) /(p-t)$ and $(s-t) /(s-p)$, we have

$$
\begin{aligned}
\int_{\xi_{1}}^{\sigma_{1}} & \int_{\xi_{2}}^{\sigma_{2}} \int_{\xi_{3}}^{\sigma_{3}}\left|h\left(\delta_{1}, \delta_{2}, \delta_{3}\right)\right|\left|f\left(\delta_{1}, \delta_{2}, \delta_{3}\right)+g\left(\delta_{1}, \delta_{2}, \delta_{3}\right)\right|^{p} \diamond \delta_{1} \diamond \delta_{2} \diamond \delta_{3} \\
\geq & \left(\int_{\xi_{1}}^{\sigma_{1}} \int_{\xi_{2}}^{\sigma_{2}} \int_{\xi_{3}}^{\sigma_{3}}\left|h\left(\delta_{1}, \delta_{2}, \delta_{3}\right)\right|\left|f\left(\delta_{1}, \delta_{2}, \delta_{3}\right)+g\left(\delta_{1}, \delta_{2}, \delta_{3}\right)\right|^{s} \diamond \delta_{1} \diamond \delta_{2} \diamond \delta_{3}\right)^{(p-t) /(s-t)} \\
& \times\left(\int_{\xi_{1}}^{\sigma_{1}} \int_{\xi_{2}}^{\sigma_{2}} \int_{\xi_{3}}^{\sigma_{3}}\left|h\left(\delta_{1}, \delta_{2}, \delta_{3}\right)\right|\left|f\left(\delta_{1}, \delta_{2}, \delta_{3}\right)+g\left(\delta_{1}, \delta_{2}, \delta_{3}\right)\right|^{t} \diamond \delta_{1} \diamond \delta_{2} \diamond \delta_{3}\right)^{(s-p) /(s-t)}
\end{aligned}
$$

On the other hand, by using Minkowski's inequality (8) for $s>1$ and $t>1$, respectively, we can see that the following assertions hold true

$$
\begin{gathered}
\left(\int_{\xi_{1}}^{\sigma_{1}} \int_{\xi_{2}}^{\sigma_{2}} \int_{\xi_{3}}^{\sigma_{3}}\left|h\left(\delta_{1}, \delta_{2}, \delta_{3}\right)\right|\left|f\left(\delta_{1}, \delta_{2}, \delta_{3}\right)+g\left(\delta_{1}, \delta_{2}, \delta_{3}\right)\right|^{s} \diamond \delta_{1} \diamond \delta_{2} \diamond \delta_{3}\right)^{\frac{1}{s}} \\
\geq\left(\int_{\xi_{1}}^{\sigma_{1}} \int_{\xi_{2}}^{\sigma_{2}} \int_{\xi_{3}}^{\sigma_{3}}\left|h\left(\delta_{1}, \delta_{2}, \delta_{3}\right)\right|\left|f\left(\delta_{1}, \delta_{2}, \delta_{3}\right)\right|^{s} \diamond \delta_{1} \diamond \delta_{2} \diamond \delta_{3}\right)^{\frac{1}{s}} \\
\quad+\left(\int_{\xi_{1}}^{\sigma_{1}} \int_{\xi_{2}}^{\sigma_{2}} \int_{\xi_{3}}^{\sigma_{3}}\left|h\left(\delta_{1}, \delta_{2}, \delta_{3}\right)\right|\left|g\left(\delta_{1}, \delta_{2}, \delta_{3}\right)\right|^{s} \diamond \delta_{1} \diamond \delta_{2} \diamond \delta_{3}\right)^{\frac{1}{s}}
\end{gathered}
$$

and

$$
\begin{gathered}
\left(\int_{\xi_{1}}^{\sigma_{1}} \int_{\xi_{2}}^{\sigma_{2}} \int_{\xi_{3}}^{\sigma_{3}}\left|h\left(\delta_{1}, \delta_{2}, \delta_{3}\right)\right|\left|f\left(\delta_{1}, \delta_{2}, \delta_{3}\right)+g\left(\delta_{1}, \delta_{2}, \delta_{3}\right)\right|^{t} \diamond \delta_{1} \diamond \delta_{2} \diamond \delta_{3}\right)^{\frac{1}{t}} \\
\geq\left(\int_{\xi_{1}}^{\sigma_{1}} \int_{\xi_{2}}^{\sigma_{2}} \int_{\xi_{3}}^{\sigma_{3}}\left|h\left(\delta_{1}, \delta_{2}, \delta_{3}\right)\right|\left|f\left(\delta_{1}, \delta_{2}, \delta_{3}\right)\right|^{t} \diamond \delta_{1} \diamond \delta_{2} \diamond \delta_{3}\right)^{\frac{1}{t}} \\
\quad+\left(\int_{\xi_{1}}^{\sigma_{1}} \int_{\xi_{2}}^{\sigma_{2}} \int_{\xi_{3}}^{\sigma_{3}}\left|h\left(\delta_{1}, \delta_{2}, \delta_{3}\right)\right|\left|g\left(\delta_{1}, \delta_{2}, \delta_{3}\right)\right|^{t} \diamond \delta_{1} \diamond \delta_{2} \diamond \delta_{3}\right)^{\frac{1}{t}}
\end{gathered}
$$

So we get the result

$$
\begin{aligned}
\int_{\xi_{1}}^{\sigma_{1}} \int_{\xi_{2}}^{\sigma_{2}} \int_{\xi_{3}}^{\sigma_{3}}\left|h\left(\delta_{1}, \delta_{2}, \delta_{3}\right)\right|\left|f\left(\delta_{1}, \delta_{2}, \delta_{3}\right)+g\left(\delta_{1}, \delta_{2}, \delta_{3}\right)\right|^{p} \diamond \delta_{1} \diamond \delta_{2} \diamond \delta_{3} \\
\geq \\
\quad\left[\left(\int_{\xi_{1}}^{\sigma_{1}} \int_{\xi_{2}}^{\sigma_{2}} \int_{\xi_{3}}^{\sigma_{3}}\left|h\left(\delta_{1}, \delta_{2}, \delta_{3}\right)\right|\left|f\left(\delta_{1}, \delta_{2}, \delta_{3}\right)\right|^{s} \diamond \delta_{1} \diamond \delta_{2} \diamond \delta_{3}\right)^{\frac{1}{s}}\right. \\
\left.+\left(\int_{\xi_{1}}^{\sigma_{1}} \int_{\xi_{2}}^{\sigma_{2}} \int_{\xi_{3}}^{\sigma_{3}}\left|h\left(\delta_{1}, \delta_{2}, \delta_{3}\right)\right|\left|g\left(\delta_{1}, \delta_{2}, \delta_{3}\right)\right|^{s} \diamond \delta_{1} \diamond \delta_{2} \diamond \delta_{3}\right)^{\frac{1}{s}}\right]^{s(p-t) /(s-t)}
\end{aligned}
$$




$$
\begin{aligned}
& \times\left[\left(\int_{\xi_{1}}^{\sigma_{1}} \int_{\xi_{2}}^{\sigma_{2}} \int_{\xi_{3}}^{\sigma_{3}}\left|h\left(\delta_{1}, \delta_{2}, \delta_{3}\right)\right|\left|f\left(\delta_{1}, \delta_{2}, \delta_{3}\right)\right|^{t} \diamond \delta_{1} \diamond \delta_{2} \diamond \delta_{3}\right)^{\frac{1}{t}}\right. \\
& \left.+\left(\int_{\xi_{1}}^{\sigma_{1}} \int_{\xi_{2}}^{\sigma_{2}} \int_{\xi_{3}}^{\sigma_{3}}\left|h\left(\delta_{1}, \delta_{2}, \delta_{3}\right)\right|\left|g\left(\delta_{1}, \delta_{2}, \delta_{3}\right)\right|^{t} \diamond \delta_{1} \diamond \delta_{2} \diamond \delta_{3}\right)^{\frac{1}{t}}\right]^{t(s-p) /(s-t)} .
\end{aligned}
$$

Remark 4.2 (1) Under the conditions of Theorem 4.1, for $p>1$, letting $s=p+\varepsilon, t=p-\varepsilon$, when $p, s, t$ are different, $s, t>1$, and letting $\varepsilon \rightarrow 0$, we obtain

$$
\begin{aligned}
\int_{\xi_{1}}^{\sigma_{1}} & \int_{\xi_{2}}^{\sigma_{2}} \int_{\xi_{3}}^{\sigma_{3}}\left|h\left(\delta_{1}, \delta_{2}, \delta_{3}\right)\right|\left|f\left(\delta_{1}, \delta_{2}, \delta_{3}\right)+g\left(\delta_{1}, \delta_{2}, \delta_{3}\right)\right|^{p} \diamond \delta_{1} \diamond \delta_{2} \diamond \delta_{3} \\
\leq & \left(\int_{\xi_{1}}^{\sigma_{1}} \int_{\xi_{2}}^{\sigma_{2}} \int_{\xi_{3}}^{\sigma_{3}}\left|h\left(\delta_{1}, \delta_{2}, \delta_{3}\right)\right|\left|f\left(\delta_{1}, \delta_{2}, \delta_{3}\right)\right|^{p} \diamond \delta_{1} \diamond \delta_{2} \diamond \delta_{3}\right)^{\frac{1}{p}} \\
& +\left(\int_{\xi_{1}}^{\sigma_{1}} \int_{\xi_{2}}^{\sigma_{2}} \int_{\xi_{3}}^{\sigma_{3}}\left|h\left(\delta_{1}, \delta_{2}, \delta_{3}\right)\right|\left|g\left(\delta_{1}, \delta_{2}, \delta_{3}\right)\right|^{p} \diamond \delta_{1} \diamond \delta_{2} \diamond \delta_{3}\right)^{\frac{1}{p}} .
\end{aligned}
$$

(2) Under the conditions of Theorem 4.1, for $0<p<1$, letting $s=p+\varepsilon, t=p-\varepsilon$, when $p, s, t$ are different, $0<s, t<1$, and letting $\varepsilon \rightarrow 0$, we obtain

$$
\begin{gathered}
\int_{\xi_{1}}^{\sigma_{1}} \int_{\xi_{2}}^{\sigma_{2}} \int_{\xi_{3}}^{\sigma_{3}}\left|h\left(\delta_{1}, \delta_{2}, \delta_{3}\right)\right|\left|f\left(\delta_{1}, \delta_{2}, \delta_{3}\right)+g\left(\delta_{1}, \delta_{2}, \delta_{3}\right)\right|^{p} \diamond \delta_{1} \diamond \delta_{2} \diamond \delta_{3} \\
\geq\left(\left.\int_{\xi_{1}}^{\sigma_{1}} \int_{\xi_{2}}^{\sigma_{2}} \int_{\xi_{3}}^{\sigma_{3}}\left|h\left(\delta_{1}, \delta_{2}, \delta_{3}\right)\right| f\left(\delta_{1}, \delta_{2}, \delta_{3}\right)\right|^{p} \diamond \delta_{1} \diamond \delta_{2} \diamond \delta_{3}\right)^{\frac{1}{p}} \\
\quad+\left(\int_{\xi_{1}}^{\sigma_{1}} \int_{\xi_{2}}^{\sigma_{2}} \int_{\xi_{3}}^{\sigma_{3}}\left|h\left(\delta_{1}, \delta_{2}, \delta_{3}\right)\right|\left|g\left(\delta_{1}, \delta_{2}, \delta_{3}\right)\right|^{p} \diamond \delta_{1} \diamond \delta_{2} \diamond \delta_{3}\right)^{\frac{1}{p}} .
\end{gathered}
$$

Now, on the basis of Theorem 4.1, we give the $n$-tuple diamond- $\alpha$ integral Minkowski's inequality on time scales.

Theorem 4.3 Let $f, g, h: \mathbb{T} \rightarrow \mathbb{R}$ be $\diamond$-integrable on $[\xi, \sigma]_{\mathbb{T}}, p>0, s, t \in \mathbb{R}$, and $s \neq t$.

(1) Let $p, s, t \in \mathbb{R}$ be different such that $s, t>1$ and $(s-t) /(p-t)>1$. Then

$$
\begin{aligned}
\int_{\xi_{1}}^{\sigma_{1}} & \int_{\xi_{2}}^{\sigma_{2}} \ldots \int_{\xi_{n}}^{\sigma_{n}}\left|h\left(\delta_{1}, \delta_{2}, \ldots, \delta_{n}\right)\right|\left|f\left(\delta_{1}, \delta_{2}, \ldots, \delta_{n}\right)+g\left(\delta_{1}, \delta_{2}, \ldots, \delta_{n}\right)\right|^{p} \diamond \delta_{1} \diamond \delta_{2} \cdots \diamond \delta_{n} \\
\leq & {\left[\left(\left.\int_{\xi_{1}}^{\sigma_{1}} \int_{\xi_{2}}^{\sigma_{2}} \cdots \int_{\xi_{n}}^{\sigma_{n}}\left|h\left(\delta_{1}, \delta_{2}, \ldots, \delta_{n}\right)\right| f\left(\delta_{1}, \delta_{2}, \ldots, \delta_{n}\right)\right|^{s} \diamond \delta_{1} \diamond \delta_{2} \cdots \diamond \delta_{n}\right)^{\frac{1}{s}}\right.} \\
& +\left(\int_{\xi_{1}}^{\sigma_{1}} \int_{\xi_{2}}^{\sigma_{2}} \cdots \int_{\xi_{n}}^{\sigma_{n}}\left|h\left(\delta_{1}, \delta_{2}, \ldots, \delta_{n}\right)\right|\right. \\
& \left.\left.\times\left|g\left(\delta_{1}, \delta_{2}, \ldots, \delta_{n}\right)\right|^{s} \diamond \delta_{1} \diamond \delta_{2} \ldots \diamond \delta_{n}\right)^{\frac{1}{s}}\right]^{s(p-t) /(s-t)} \\
& \times\left[\left(\int_{\xi_{1}}^{\sigma_{1}} \int_{\xi_{2}}^{\sigma_{2}} \ldots \int_{\xi_{n}}^{\sigma_{n}}\left|h\left(\delta_{1}, \delta_{2}, \ldots, \delta_{n}\right)\right|\left|f\left(\delta_{1}, \delta_{2}, \ldots, \delta_{n}\right)\right|^{t} \diamond \delta_{1} \diamond \delta_{2} \cdots \nabla \delta_{n}\right)^{\frac{1}{t}}\right. \\
& +\left(\int_{\xi_{1}}^{\sigma_{1}} \int_{\xi_{2}}^{\sigma_{2}} \ldots \int_{\xi_{n}}^{\sigma_{n}}\left|h\left(\delta_{1}, \delta_{2}, \ldots, \delta_{n}\right)\right|\right.
\end{aligned}
$$




$$
\left.\left.\times\left|g\left(\delta_{1}, \delta_{2}, \ldots, \delta_{n}\right)\right|^{t} \diamond \delta_{1} \diamond \delta_{2} \ldots \diamond \delta_{n}\right)^{\frac{1}{t}}\right]^{t(s-p) /(s-t)}
$$

(2) Let $p, s, t \in \mathbb{R}$ be different such that $0<s<1,0<t<1$ and $(s-t) /(p-t)<1$. Then

$$
\begin{aligned}
& \int_{\xi_{1}}^{\sigma_{1}} \int_{\xi_{2}}^{\sigma_{2}} \cdots \int_{\xi_{n}}^{\sigma_{n}}\left|h\left(\delta_{1}, \delta_{2}, \ldots, \delta_{n}\right)\right|\left|f\left(\delta_{1}, \delta_{2}, \ldots, \delta_{n}\right)+g\left(\delta_{1}, \delta_{2}, \ldots, \delta_{n}\right)\right|^{p} \diamond \delta_{1} \diamond \delta_{2} \cdots \diamond \delta_{n} \\
& \geq\left[\left(\int_{\xi_{1}}^{\sigma_{1}} \int_{\xi_{2}}^{\sigma_{2}} \cdots \int_{\xi_{n}}^{\sigma_{n}}\left|h\left(\delta_{1}, \delta_{2}, \ldots, \delta_{n}\right)\right|\left|f\left(\delta_{1}, \delta_{2}, \ldots, \delta_{n}\right)\right|^{s} \diamond \delta_{1} \diamond \delta_{2} \cdots \diamond \delta_{n}\right)^{\frac{1}{s}}\right. \\
& +\left(\int_{\xi_{1}}^{\sigma_{1}} \int_{\xi_{2}}^{\sigma_{2}} \cdots \int_{\xi_{n}}^{\sigma_{n}}\left|h\left(\delta_{1}, \delta_{2}, \ldots, \delta_{n}\right)\right|\right. \\
& \left.\left.\times\left|g\left(\delta_{1}, \delta_{2}, \ldots, \delta_{n}\right)\right|^{s} \diamond \delta_{1} \diamond \delta_{2} \cdots \diamond \delta_{n}\right)^{\frac{1}{s}}\right]^{s(p-t) /(s-t)} \\
& \times\left[\left(\int_{\xi_{1}}^{\sigma_{1}} \int_{\xi_{2}}^{\sigma_{2}} \ldots \int_{\xi_{n}}^{\sigma_{n}}\left|h\left(\delta_{1}, \delta_{2}, \ldots, \delta_{n}\right)\right|\left|f\left(\delta_{1}, \delta_{2}, \ldots, \delta_{n}\right)\right|^{t} \diamond \delta_{1} \diamond \delta_{2} \ldots \diamond \delta_{n}\right)^{\frac{1}{t}}\right. \\
& +\left(\int_{\xi_{1}}^{\sigma_{1}} \int_{\xi_{2}}^{\sigma_{2}} \cdots \int_{\xi_{n}}^{\sigma_{n}}\left|h\left(\delta_{1}, \delta_{2}, \ldots, \delta_{n}\right)\right|\right. \\
& \left.\left.\times\left|g\left(\delta_{1}, \delta_{2}, \ldots, \delta_{n}\right)\right|^{t} \diamond \delta_{1} \diamond \delta_{2} \ldots \diamond \delta_{n}\right)^{\frac{1}{t}}\right]^{t(s-p) /(s-t)} .
\end{aligned}
$$

Proof Similar to the proof of Theorem 4.1, we get the result of Theorem 4.3.

Remark 4.4 Aiming at the diamond- $\alpha$ integral Minkowski's inequality proposed by Theorem 3.5 in Ref. [17], we generalize it in this paper and obtain the three-tuple and $n$-tuple diamond- $\alpha$ inequalities (34)-(37).

Theorem 4.5 Let $f, g, h: \mathbb{T} \rightarrow \mathbb{R}$ and $0<r<1<p$.Iff, $g$ and $h$ are $\diamond$-integrable on $[\xi, \sigma]_{\mathbb{T}}$, then

$$
\begin{aligned}
& \left(\frac{\int_{\xi_{1}}^{\sigma_{1}} \int_{\xi_{2}}^{\sigma_{2}} \int_{\xi_{3}}^{\sigma_{3}}\left|h\left(\delta_{1}, \delta_{2}, \delta_{3}\right)\right|\left|f\left(\delta_{1}, \delta_{2}, \delta_{3}\right)+g\left(\delta_{1}, \delta_{2}, \delta_{3}\right)\right|^{p} \diamond \delta_{1} \diamond \delta_{2} \diamond \delta_{3}}{\int_{\xi_{1}}^{\sigma_{1}} \int_{\xi_{2}}^{\sigma_{2}} \int_{\xi_{3}}^{\sigma_{3}}\left|h\left(\delta_{1}, \delta_{2}, \delta_{3}\right)\right|\left|f\left(\delta_{1}, \delta_{2}, \delta_{3}\right)+g\left(\delta_{1}, \delta_{2}, \delta_{3}\right)\right|^{r} \diamond \delta_{1} \diamond \delta_{2} \diamond \delta_{3}}\right)^{1 /(p-r)} \\
& \leq\left(\frac{\int_{\xi_{1}}^{\sigma_{1}} \int_{\xi_{2}}^{\sigma_{2}} \int_{\xi_{3}}^{\sigma_{3}}\left|h\left(\delta_{1}, \delta_{2}, \delta_{3}\right)\right|\left|f\left(\delta_{1}, \delta_{2}, \delta_{3}\right)\right|^{p} \diamond \delta_{1} \diamond \delta_{2} \diamond \delta_{3}}{\int_{\xi_{1}}^{\sigma_{1}} \int_{\xi_{2}}^{\sigma_{2}} \int_{\xi_{3}}^{\sigma_{3}}\left|h\left(\delta_{1}, \delta_{2}, \delta_{3}\right)\right|\left|f\left(\delta_{1}, \delta_{2}, \delta_{3}\right)\right|^{r} \diamond \delta_{1} \diamond \delta_{2} \diamond \delta_{3}}\right)^{1 /(p-r)} \\
& +\left(\frac{\int_{\xi_{1}}^{\sigma_{1}} \int_{\xi_{2}}^{\sigma_{2}} \int_{\xi_{3}}^{\sigma_{3}}\left|h\left(\delta_{1}, \delta_{2}, \delta_{3}\right)\right|\left|g\left(\delta_{1}, \delta_{2}, \delta_{3}\right)\right|^{p} \diamond \delta_{1} \diamond \delta_{2} \diamond \delta_{3}}{\int_{\xi_{1}}^{\sigma_{1}} \int_{\xi_{2}}^{\sigma_{2}} \int_{\xi_{3}}^{\sigma_{3}}\left|h\left(\delta_{1}, \delta_{2}, \delta_{3}\right)\right|\left|g\left(\delta_{1}, \delta_{2}, \delta_{3}\right)\right|^{r} \diamond \delta_{1} \diamond \delta_{2} \diamond \delta_{3}}\right)^{1 /(p-r)} .
\end{aligned}
$$

Proof From inequality (5) and inequality (6), we have

$$
\begin{gathered}
\left(\int_{\xi_{1}}^{\sigma_{1}} \int_{\xi_{2}}^{\sigma_{2}} \int_{\xi_{3}}^{\sigma_{3}}\left|h\left(\delta_{1}, \delta_{2}, \delta_{3}\right)\right|\left|f\left(\delta_{1}, \delta_{2}, \delta_{3}\right)+g\left(\delta_{1}, \delta_{2}, \delta_{3}\right)\right|^{p} \diamond \delta_{1} \diamond \delta_{2} \diamond \delta_{3}\right)^{1 /(p-r)} \\
\leq\left(\left(\int_{\xi_{1}}^{\sigma_{1}} \int_{\xi_{2}}^{\sigma_{2}} \int_{\xi_{3}}^{\sigma_{3}}\left|h\left(\delta_{1}, \delta_{2}, \delta_{3}\right)\right|\left|f\left(\delta_{1}, \delta_{2}, \delta_{3}\right)\right|^{p} \diamond \delta_{1} \diamond \delta_{2} \diamond \delta_{3}\right)^{1 / p}\right. \\
\left.\quad+\left(\int_{\xi_{1}}^{\sigma_{1}} \int_{\xi_{2}}^{\sigma_{2}} \int_{\xi_{3}}^{\sigma_{3}}\left|h\left(\delta_{1}, \delta_{2}, \delta_{3}\right)\right|\left|g\left(\delta_{1}, \delta_{2}, \delta_{3}\right)\right|^{p} \diamond \delta_{1} \diamond \delta_{2} \diamond \delta_{3}\right)^{1 / p}\right)^{p /(p-r)}
\end{gathered}
$$




$$
\begin{aligned}
& =\left(\left(\frac{\int_{\xi_{1}}^{\sigma_{1}} \int_{\xi_{2}}^{\sigma_{2}} \int_{\xi_{3}}^{\sigma_{3}}\left|h\left(\delta_{1}, \delta_{2}, \delta_{3}\right)\right|\left|f\left(\delta_{1}, \delta_{2}, \delta_{3}\right)\right|^{p} \diamond \delta_{1} \diamond \delta_{2} \diamond \delta_{3}}{\int_{\xi_{1}}^{\sigma_{1}} \int_{\xi_{2}}^{\sigma_{2}} \int_{\xi_{3}}^{\sigma_{3}}\left|h\left(\delta_{1}, \delta_{2}, \delta_{3}\right)\right|\left|f\left(\delta_{1}, \delta_{2}, \delta_{3}\right)\right|^{r} \diamond \delta_{1} \diamond \delta_{2} \diamond \delta_{3}}\right)^{1 / p}\right. \\
& \times\left(\int_{\xi_{1}}^{\sigma_{1}} \int_{\xi_{2}}^{\sigma_{2}} \int_{\xi_{3}}^{\sigma_{3}}\left|h\left(\delta_{1}, \delta_{2}, \delta_{3}\right)\right|\left|f\left(\delta_{1}, \delta_{2}, \delta_{3}\right)\right|^{r} \diamond \delta_{1} \diamond \delta_{2} \diamond \delta_{3}\right)^{1 / p} \\
& +\left(\frac{\int_{\xi_{1}}^{\sigma_{1}} \int_{\xi_{2}}^{\sigma_{2}} \int_{\xi_{3}}^{\sigma_{3}}\left|h\left(\delta_{1}, \delta_{2}, \delta_{3}\right)\right|\left|g\left(\delta_{1}, \delta_{2}, \delta_{3}\right)\right|^{p} \diamond \delta_{1} \diamond \delta_{2} \diamond \delta_{3}}{\int_{\xi_{1}}^{\sigma_{1}} \int_{\xi_{2}}^{\sigma_{2}} \int_{\xi_{3}}^{\sigma_{3}}\left|h\left(\delta_{1}, \delta_{2}, \delta_{3}\right)\right|\left|g\left(\delta_{1}, \delta_{2}, \delta_{3}\right)\right|^{r} \diamond \delta_{1} \diamond \delta_{2} \diamond \delta_{3}}\right)^{1 / p} \\
& \left.\times\left(\int_{\xi_{1}}^{\sigma_{1}} \int_{\xi_{2}}^{\sigma_{2}} \int_{\xi_{3}}^{\sigma_{3}}\left|h\left(\delta_{1}, \delta_{2}, \delta_{3}\right)\right|\left|g\left(\delta_{1}, \delta_{2}, \delta_{3}\right)\right|^{r} \diamond \delta_{1} \diamond \delta_{2} \diamond \delta_{3}\right)^{1 / p}\right)^{p /(p-r)} \\
& \leq\left(\left(\frac{\int_{\xi_{1}}^{\sigma_{1}} \int_{\xi_{2}}^{\sigma_{2}} \int_{\xi_{3}}^{\sigma_{3}}\left|h\left(\delta_{1}, \delta_{2}, \delta_{3}\right)\right|\left|f\left(\delta_{1}, \delta_{2}, \delta_{3}\right)\right|^{p} \diamond \delta_{1} \diamond \delta_{2} \diamond \delta_{3}}{\int_{\xi_{1}}^{\sigma_{1}} \int_{\xi_{2}}^{\sigma_{2}} \int_{\xi_{3}}^{\sigma_{3}}\left|h\left(\delta_{1}, \delta_{2}, \delta_{3}\right)\right|\left|f\left(\delta_{1}, \delta_{2}, \delta_{3}\right)\right|^{r} \diamond \delta_{1} \diamond \delta_{2} \diamond \delta_{3}}\right)^{1 /(p-r)}\right. \\
& \left.+\left(\frac{\int_{\xi_{1}}^{\sigma_{1}} \int_{\xi_{2}}^{\sigma_{2}} \int_{\xi_{3}}^{\sigma_{3}}\left|h\left(\delta_{1}, \delta_{2}, \delta_{3}\right)\right|\left|g\left(\delta_{1}, \delta_{2}, \delta_{3}\right)\right|^{p} \diamond \delta_{1} \diamond \delta_{2} \diamond \delta_{3}}{\int_{\xi_{1}}^{\sigma_{1}} \int_{\xi_{2}}^{\sigma_{2}} \int_{\xi_{3}}^{\sigma_{3}}\left|h\left(\delta_{1}, \delta_{2}, \delta_{3}\right)\right|\left|g\left(\delta_{1}, \delta_{2}, \delta_{3}\right)\right|^{r} \diamond \delta_{1} \diamond \delta_{2} \diamond \delta_{3}}\right)^{1 /(p-r)}\right) \\
& \times\left(\left(\int_{\xi_{1}}^{\sigma_{1}} \int_{\xi_{2}}^{\sigma_{2}} \int_{\xi_{3}}^{\sigma_{3}}\left|h\left(\delta_{1}, \delta_{2}, \delta_{3}\right)\right|\left|f\left(\delta_{1}, \delta_{2}, \delta_{3}\right)\right|^{r} \diamond \delta_{1} \diamond \delta_{2} \diamond \delta_{3}\right)^{1 / r}\right. \\
& \left.+\left(\int_{\xi_{1}}^{\sigma_{1}} \int_{\xi_{2}}^{\sigma_{2}} \int_{\xi_{3}}^{\sigma_{3}}\left|h\left(\delta_{1}, \delta_{2}, \delta_{3}\right)\right|\left|g\left(\delta_{1}, \delta_{2}, \delta_{3}\right)\right|^{r} \diamond \delta_{1} \diamond \delta_{2} \diamond \delta_{3}\right)^{1 / r}\right)^{r /(p-r)} .
\end{aligned}
$$

From inequality (6), we get

$$
\begin{aligned}
& \left(\left(\int_{\xi_{1}}^{\sigma_{1}} \int_{\xi_{2}}^{\sigma_{2}} \int_{\xi_{3}}^{\sigma_{3}}\left|h\left(\delta_{1}, \delta_{2}, \delta_{3}\right)\right|\left|f\left(\delta_{1}, \delta_{2}, \delta_{3}\right)\right|^{r} \diamond \delta_{1} \diamond \delta_{2} \diamond \delta_{3}\right)^{1 / r}\right. \\
& \left.\quad+\left(\int_{\xi_{1}}^{\sigma_{1}} \int_{\xi_{2}}^{\sigma_{2}} \int_{\xi_{3}}^{\sigma_{3}}\left|h\left(\delta_{1}, \delta_{2}, \delta_{3}\right)\right|\left|g\left(\delta_{1}, \delta_{2}, \delta_{3}\right)\right|^{r} \diamond \delta_{1} \diamond \delta_{2} \diamond \delta_{3}\right)^{1 / r}\right)^{r} \\
& \quad \leq \int_{\xi_{1}}^{\sigma_{1}} \int_{\xi_{2}}^{\sigma_{2}} \int_{\xi_{3}}^{\sigma_{3}}\left|h\left(\delta_{1}, \delta_{2}, \delta_{3}\right)\right|\left|f\left(\delta_{1}, \delta_{2}, \delta_{3}\right)+g\left(\delta_{1}, \delta_{2}, \delta_{3}\right)\right|^{r} \diamond \delta_{1} \diamond \delta_{2} \diamond \delta_{3} .
\end{aligned}
$$

Hence, we have

$$
\begin{gathered}
\left(\frac{\int_{\xi_{1}}^{\sigma_{1}} \int_{\xi_{2}}^{\sigma_{2}} \int_{\xi_{3}}^{\sigma_{3}}\left|h\left(\delta_{1}, \delta_{2}, \delta_{3}\right)\right|\left|f\left(\delta_{1}, \delta_{2}, \delta_{3}\right)+g\left(\delta_{1}, \delta_{2}, \delta_{3}\right)\right|^{p} \diamond \delta_{1} \diamond \delta_{2} \diamond \delta_{3}}{\int_{\xi_{1}}^{\sigma_{1}} \int_{\xi_{2}}^{\sigma_{2}} \int_{\xi_{3}}^{\sigma_{3}}\left|h\left(\delta_{1}, \delta_{2}, \delta_{3}\right)\right|\left|f\left(\delta_{1}, \delta_{2}, \delta_{3}\right)+g\left(\delta_{1}, \delta_{2}, \delta_{3}\right)\right|^{r} \diamond \delta_{1} \diamond \delta_{2} \diamond \delta_{3}}\right)^{1 /(p-r)} \\
\leq\left(\frac{\int_{\xi_{1}}^{\sigma_{1}} \int_{\xi_{2}}^{\sigma_{2}} \int_{\xi_{3}}^{\sigma_{3}}\left|h\left(\delta_{1}, \delta_{2}, \delta_{3}\right)\right|\left|f\left(\delta_{1}, \delta_{2}, \delta_{3}\right)\right|^{p} \diamond \delta_{1} \diamond \delta_{2} \diamond \delta_{3}}{\int_{\xi_{1}}^{\sigma_{1}} \int_{\xi_{2}}^{\sigma_{2}} \int_{\xi_{3}}^{\sigma_{3}}\left|h\left(\delta_{1}, \delta_{2}, \delta_{3}\right)\right|\left|f\left(\delta_{1}, \delta_{2}, \delta_{3}\right)\right|^{r} \diamond \delta_{1} \diamond \delta_{2} \diamond \delta_{3}}\right)^{1 /(p-r)} \\
\quad+\left(\frac{\int_{\xi_{1}}^{\sigma_{1}} \int_{\xi_{2}}^{\sigma_{2}} \int_{\xi_{3}}^{\sigma_{3}}\left|h\left(\delta_{1}, \delta_{2}, \delta_{3}\right)\right|\left|g\left(\delta_{1}, \delta_{2}, \delta_{3}\right)\right|^{p} \diamond \delta_{1} \diamond \delta_{2} \diamond \delta_{3}}{\int_{\xi_{1}}^{\sigma_{1}} \int_{\xi_{2}}^{\sigma_{2}} \int_{\xi_{3}}^{\sigma_{3}}\left|h\left(\delta_{1}, \delta_{2}, \delta_{3}\right)\right|\left|g\left(\delta_{1}, \delta_{2}, \delta_{3}\right)\right|^{r} \diamond \delta_{1} \diamond \delta_{2} \diamond \delta_{3}}\right)^{1 /(p-r)} .
\end{gathered}
$$

The proof of Theorem 4.5 is completed.

Next, on the basis of Theorem 4.5, we give the $n$-tuple diamond- $\alpha$ integral Minkowski's inequality on time scales. 
Theorem 4.6 Let $f, g, h: \mathbb{T} \rightarrow \mathbb{R}$ and $0<r<1<p$.Iff, $g$ and $h$ are $\diamond$-integrable on $[\xi, \sigma]_{\mathbb{T}}$, then

$$
\begin{aligned}
& \left(\frac{\int_{\xi_{1}}^{\sigma_{1}} \int_{\xi_{2}}^{\sigma_{2}} \cdots \int_{\xi_{n}}^{\sigma_{n}}\left|h\left(\delta_{1}, \delta_{2}, \ldots, \delta_{n}\right)\right|\left|f\left(\delta_{1}, \delta_{2}, \ldots, \delta_{n}\right)+g\left(\delta_{1}, \delta_{2}, \ldots, \delta_{n}\right)\right|^{p} \diamond \delta_{1} \diamond \delta_{2} \cdots \diamond \delta_{n}}{\int_{\xi_{1}}^{\sigma_{1}} \int_{\xi_{2}}^{\sigma_{2}} \cdots \int_{\xi_{n}}^{\sigma_{n}}\left|h\left(\delta_{1}, \delta_{2}, \ldots, \delta_{n}\right)\right|\left|f\left(\delta_{1}, \delta_{2}, \ldots, \delta_{n}\right)+g\left(\delta_{1}, \delta_{2}, \ldots, \delta_{n}\right)\right|^{r} \diamond \delta_{1} \diamond \delta_{2} \cdots \diamond \delta_{n}}\right)^{1 /(p-r)} \\
& \leq\left(\frac{\left.\int_{\xi_{1}}^{\sigma_{1}} \int_{\xi_{2}}^{\sigma_{2}} \cdots \int_{\xi_{n}}^{\sigma_{n}}\left|h\left(\delta_{1}, \delta_{2}, \ldots, \delta_{n}\right)\right| f\left(\delta_{1}, \delta_{2}, \ldots, \delta_{n}\right)\right|^{p} \diamond \delta_{1} \diamond \delta_{2} \cdots \diamond \delta_{n}}{\left.\int_{\xi_{1}}^{\sigma_{1}} \int_{\xi_{2}}^{\sigma_{2}} \cdots \int_{\xi_{n}}^{\sigma_{n}}\left|h\left(\delta_{1}, \delta_{2}, \ldots, \delta_{n}\right)\right| f\left(\delta_{1}, \delta_{2}, \ldots, \delta_{n}\right)\right|^{r} \diamond \delta_{1} \diamond \delta_{2} \cdots \diamond \delta_{n}}\right)^{1 /(p-r)} \\
& +\left(\frac{\int_{\xi_{1}}^{\sigma_{1}} \int_{\xi_{2}}^{\sigma_{2}} \cdots \int_{\xi_{n}}^{\sigma_{n}}\left|h\left(\delta_{1}, \delta_{2}, \ldots, \delta_{n}\right)\right|\left|g\left(\delta_{1}, \delta_{2}, \ldots, \delta_{n}\right)\right|^{p} \diamond \delta_{1} \diamond \delta_{2} \cdots \diamond \delta_{3}}{\int_{\xi_{1}}^{\sigma_{1}} \int_{\xi_{2}}^{\sigma_{2}} \cdots \int_{\xi_{n}}^{\sigma_{n}}\left|h\left(\delta_{1}, \delta_{2}, \ldots, \delta_{n}\right)\right|\left|g\left(\delta_{1}, \delta_{2}, \ldots, \delta_{n}\right)\right|^{r} \diamond \delta_{1} \oslash \delta_{2} \cdots \diamond \delta_{n}}\right)^{1 /(p-r)} .
\end{aligned}
$$

Proof Similar to the proof of Theorem 4.5, we get the result of Theorem 4.6.

Remark 4.7 The inequalities in Theorem 4.5 and Theorem 4.6 are generalized results for Theorem 3.6 in Ref. [17].

Theorem 4.8 Let $f, g, h: \mathbb{T} \rightarrow \mathbb{R}$ and $p \leq 0 \leq r$. If $f, g, f^{p}, g^{p}$ and $h$ are $\diamond$-integrable on $[\xi, \sigma]_{\mathbb{T}}$, then

$$
\begin{aligned}
& \left(\frac{\int_{\xi_{1}}^{\sigma_{1}} \int_{\xi_{2}}^{\sigma_{2}} \int_{\xi_{3}}^{\sigma_{3}}\left|h\left(\delta_{1}, \delta_{2}, \delta_{3}\right)\right|\left|f\left(\delta_{1}, \delta_{2}, \delta_{3}\right)+g\left(\delta_{1}, \delta_{2}, \delta_{3}\right)\right|^{p} \diamond \delta_{1} \diamond \delta_{2} \diamond \delta_{3}}{\int_{\xi_{1}}^{\sigma_{1}} \int_{\xi_{2}}^{\sigma_{2}} \int_{\xi_{3}}^{\sigma_{3}}\left|h\left(\delta_{1}, \delta_{2}, \delta_{3}\right)\right|\left|f\left(\delta_{1}, \delta_{2}, \delta_{3}\right)+g\left(\delta_{1}, \delta_{2}, \delta_{3}\right)\right|^{r} \diamond \delta_{1} \diamond \delta_{2} \diamond \delta_{3}}\right)^{1 /(p-r)} \\
& \geq\left(\frac{\int_{\xi_{1}}^{\sigma_{1}} \int_{\xi_{2}}^{\sigma_{2}} \int_{\xi_{3}}^{\sigma_{3}}\left|h\left(\delta_{1}, \delta_{2}, \delta_{3}\right)\right|\left|f\left(\delta_{1}, \delta_{2}, \delta_{3}\right)\right|^{p} \diamond \delta_{1} \diamond \delta_{2} \diamond \delta_{3}}{\int_{\xi_{1}}^{\sigma_{1}} \int_{\xi_{2}}^{\sigma_{2}} \int_{\xi_{3}}^{\sigma_{3}}\left|h\left(\delta_{1}, \delta_{2}, \delta_{3}\right)\right|\left|f\left(\delta_{1}, \delta_{2}, \delta_{3}\right)\right|^{r} \diamond \delta_{1} \diamond \delta_{2} \diamond \delta_{3}}\right)^{1 /(p-r)} \\
& +\left(\frac{\int_{\xi_{1}}^{\sigma_{1}} \int_{\xi_{2}}^{\sigma_{2}} \int_{\xi_{3}}^{\sigma_{3}}\left|h\left(\delta_{1}, \delta_{2}, \delta_{3}\right)\right|\left|g\left(\delta_{1}, \delta_{2}, \delta_{3}\right)\right|^{p} \diamond \delta_{1} \diamond \delta_{2} \oslash \delta_{3}}{\int_{\xi_{1}}^{\sigma_{1}} \int_{\xi_{2}}^{\sigma_{2}} \int_{\xi_{3}}^{\sigma_{3}}\left|h\left(\delta_{1}, \delta_{2}, \delta_{3}\right)\right|\left|g\left(\delta_{1}, \delta_{2}, \delta_{3}\right)\right|^{r} \diamond \delta_{1} \diamond \delta_{2} \diamond \delta_{3}}\right)^{1 /(p-r)} .
\end{aligned}
$$

Proof Let $\alpha_{1} \geq 0, \alpha_{2} \geq 0, \beta_{1}>0$, and $\beta_{2}>0$, and $-1<\lambda<0$, using the following Radon inequality:

$$
\sum_{k=1}^{n} \frac{a_{k}^{p}}{b_{k}^{p-1}} \leq \frac{\left(\sum_{k=1}^{n} a_{k}\right)^{p}}{\left(\sum_{k=1}^{n} b_{k}\right)^{p-1}}, \quad a_{k} \geq 0, b_{k}>0,0<p<1,
$$

we have

$$
\frac{\alpha_{1}^{\lambda+1}}{\beta_{1}^{\lambda}}+\frac{\alpha_{2}^{\lambda+1}}{\beta_{2}^{\lambda}} \leq \frac{\left(\alpha_{1}+\alpha_{2}\right)^{\lambda+1}}{\left(\beta_{1}+\beta_{2}\right)^{\lambda}} .
$$

Let

$$
\begin{aligned}
& \alpha_{1}=\left(\left.\int_{\xi_{1}}^{\sigma_{1}} \int_{\xi_{2}}^{\sigma_{2}} \int_{\xi_{3}}^{\sigma_{3}}\left|h\left(\delta_{1}, \delta_{2}, \delta_{3}\right)\right| f\right|^{p} \diamond \delta_{1} \diamond \delta_{2} \diamond \delta_{3}\right)^{1 / p}, \\
& \beta_{1}=\left(\int_{\xi_{1}}^{\sigma_{1}} \int_{\xi_{2}}^{\sigma_{2}} \int_{\xi_{3}}^{\sigma_{3}}\left|h\left(\delta_{1}, \delta_{2}, \delta_{3}\right)\right||f|^{r} \diamond \delta_{1} \diamond \delta_{2} \diamond \delta_{3}\right)^{1 / r}, \\
& \alpha_{2}=\left(\int_{\xi_{1}}^{\sigma_{1}} \int_{\xi_{2}}^{\sigma_{2}} \int_{\xi_{3}}^{\sigma_{3}}\left|h\left(\delta_{1}, \delta_{2}, \delta_{3}\right)\right||g|^{p} \diamond \delta_{1} \diamond \delta_{2} \diamond \delta_{3}\right)^{1 / p}, \\
& \beta_{2}=\left(\int_{\xi_{1}}^{\sigma_{1}} \int_{\xi_{2}}^{\sigma_{2}} \int_{\xi_{3}}^{\sigma_{3}}\left|h\left(\delta_{1}, \delta_{2}, \delta_{3}\right)\right||g|^{r} \diamond \delta_{1} \diamond \delta_{2} \diamond \delta_{3}\right)^{1 / r},
\end{aligned}
$$


and let $\lambda=\frac{r}{p-r}$, it follows that

$$
\begin{aligned}
& \frac{\alpha_{1}^{\lambda+1}}{\beta_{1}^{\lambda}}+\frac{\alpha_{2}^{\lambda+1}}{\beta_{2}^{\lambda}} \\
& =\frac{\left(\int_{\xi_{1}}^{\sigma_{1}} \int_{\xi_{2}}^{\sigma_{2}} \int_{\xi_{3}}^{\sigma_{3}}\left|h\left(\delta_{1}, \delta_{2}, \delta_{3}\right)\right||f|^{\triangleright} \diamond \delta_{1} \diamond \delta_{2} \oslash \delta_{3}\right)^{(\lambda+1) / p}}{\left(\int_{\xi_{1}}^{\sigma_{1}} \int_{\xi_{2}}^{\sigma_{2}} \int_{\xi_{3}}^{\sigma_{3}}\left|h\left(\delta_{1}, \delta_{2}, \delta_{3}\right)\right||f|^{r} \diamond \delta_{1} \diamond \delta_{2} \oslash \delta_{3}\right)^{\lambda / r}} \\
& +\frac{\left(\int_{\xi_{1}}^{\sigma_{1}} \int_{\xi_{2}}^{\sigma_{2}} \int_{\xi_{3}}^{\sigma_{3}}\left|h\left(\delta_{1}, \delta_{2}, \delta_{3}\right)\right||g|^{p} \diamond \delta_{1} \diamond \delta_{2} \diamond \delta_{3}\right)^{(\lambda+1) / p}}{\left(\int_{\xi_{1}}^{\sigma_{1}} \int_{\xi_{2}}^{\sigma_{2}} \int_{\xi_{3}}^{\sigma_{3}}\left|h\left(\delta_{1}, \delta_{2}, \delta_{3}\right)\right||g|^{r} \diamond \delta_{1} \diamond \delta_{2} \diamond \delta_{3}\right)^{\lambda / r}} \\
& =\left(\frac{\int_{\xi_{1}}^{\sigma_{1}} \int_{\xi_{2}}^{\sigma_{2}} \int_{\xi_{3}}^{\sigma_{3}}\left|h\left(\delta_{1}, \delta_{2}, \delta_{3}\right)\right||f|^{p} \diamond \delta_{1} \diamond \delta_{2} \diamond \delta_{3}}{\int_{\xi_{1}}^{\sigma_{1}} \int_{\xi_{2}}^{\sigma_{2}} \int_{\xi_{3}}^{\sigma_{3}}\left|h\left(\delta_{1}, \delta_{2}, \delta_{3}\right)\right||f|^{r} \diamond \delta_{1} \diamond \delta_{2} \diamond \delta_{3}}\right)^{1 /(p-r)} \\
& +\left(\frac{\int_{\xi_{1}}^{\sigma_{1}} \int_{\xi_{2}}^{\sigma_{2}} \int_{\xi_{3}}^{\sigma_{3}}\left|h\left(\delta_{1}, \delta_{2}, \delta_{3}\right)\right||g|^{p} \diamond \delta_{1} \diamond \delta_{2} \diamond \delta_{3}}{\int_{\xi_{1}}^{\sigma_{1}} \int_{\xi_{2}}^{\sigma_{2}} \int_{\xi_{3}}^{\sigma_{3}}\left|h\left(\delta_{1}, \delta_{2}, \delta_{3}\right)\right||g|^{r} \diamond \delta_{1} \diamond \delta_{2} \diamond \delta_{3}}\right)^{1 /(p-r)} \\
& \leq \frac{\left(\alpha_{1}+\alpha_{2}\right)^{\lambda+1}}{\left(\beta_{1}+\beta_{2}\right)^{\lambda}} \\
& =\left[\left(\int_{\xi_{1}}^{\sigma_{1}} \int_{\xi_{2}}^{\sigma_{2}} \int_{\xi_{3}}^{\sigma_{3}}\left|h\left(\delta_{1}, \delta_{2}, \delta_{3}\right)\right||f|^{p} \diamond \delta_{1} \diamond \delta_{2} \diamond \delta_{3}\right)^{1 / p}\right. \\
& \left.+\left(\int_{\xi_{1}}^{\sigma_{1}} \int_{\xi_{2}}^{\sigma_{2}} \int_{\xi_{3}}^{\sigma_{3}}\left|h\left(\delta_{1}, \delta_{2}, \delta_{3}\right)\right||g|^{p} \diamond \delta_{1} \diamond \delta_{2} \diamond \delta_{3}\right)^{1 / p}\right]^{p /(p-r)} \\
& /\left[\left(\int_{\xi_{1}}^{\sigma_{1}} \int_{\xi_{2}}^{\sigma_{2}} \int_{\xi_{3}}^{\sigma_{3}}\left|h\left(\delta_{1}, \delta_{2}, \delta_{3}\right)\right||f|^{r} \diamond \delta_{1} \diamond \delta_{2} \diamond \delta_{3}\right)^{1 / r}\right. \\
& \left.+\left(\int_{\xi_{1}}^{\sigma_{1}} \int_{\xi_{2}}^{\sigma_{2}} \int_{\xi_{3}}^{\sigma_{3}}\left|h\left(\delta_{1}, \delta_{2}, \delta_{3}\right)\right||g|^{r} \diamond \delta_{1} \diamond \delta_{2} \diamond \delta_{3}\right)^{1 / r}\right]^{r /(p-r)} .
\end{aligned}
$$

Since $-1<\lambda=\frac{r}{p-r}<0$, we may assume $p<0<r$, and $0<r \leq 1$, we obtain

$$
\begin{aligned}
& \quad\left[\left(\left.\int_{\xi_{1}}^{\sigma_{1}} \int_{\xi_{2}}^{\sigma_{2}} \int_{\xi_{3}}^{\sigma_{3}}\left|h\left(\delta_{1}, \delta_{2}, \delta_{3}\right)\right| f\right|^{r} \diamond \delta_{1} \diamond \delta_{2} \diamond \delta_{3}\right)^{1 / r}\right. \\
& \left.\quad+\left(\int_{\xi_{1}}^{\sigma_{1}} \int_{\xi_{2}}^{\sigma_{2}} \int_{\xi_{3}}^{\sigma_{3}}\left|h\left(\delta_{1}, \delta_{2}, \delta_{3}\right)\right||g|^{r} \diamond \delta_{1} \diamond \delta_{2} \diamond \delta_{3}\right)^{1 / r}\right]^{r} \\
& \quad \leq \int_{\xi_{1}}^{\sigma_{1}} \int_{\xi_{2}}^{\sigma_{2}} \int_{\xi_{3}}^{\sigma_{3}}\left|h\left(\delta_{1}, \delta_{2}, \delta_{3}\right)\right| f+\left.g\right|^{r} \diamond \delta_{1} \diamond \delta_{2} \diamond \delta_{3} .
\end{aligned}
$$

For $p<0$, we obtain

$$
\sum_{k=1}^{n} m_{k}^{p} n_{k}^{1 / p-1} \geq\left(\sum_{k=1}^{n} m_{k}\right)^{p}\left(\sum_{k=1}^{n} n_{k}^{1 / p}\right)^{1-p} .
$$

Assume that $f(\delta)$ and $g(\delta)$ are nonzero, let

$$
\begin{aligned}
& M=\int_{\xi_{1}}^{\sigma_{1}} \int_{\xi_{2}}^{\sigma_{2}} \int_{\xi_{3}}^{\sigma_{3}}\left|h\left(\delta_{1}, \delta_{2}, \delta_{3}\right)\right|\left|f\left(\delta_{1}, \delta_{2}, \delta_{3}\right)\right|^{p} \diamond \delta_{1} \diamond \delta_{2} \diamond \delta_{3}, \\
& N=\int_{\xi_{1}}^{\sigma_{1}} \int_{\xi_{2}}^{\sigma_{2}} \int_{\xi_{3}}^{\sigma_{3}}\left|h\left(\delta_{1}, \delta_{2}, \delta_{3}\right)\right|\left|g\left(\delta_{1}, \delta_{2}, \delta_{3}\right)\right|^{p} \diamond \delta_{1} \diamond \delta_{2} \diamond \delta_{3},
\end{aligned}
$$




$$
\begin{aligned}
W= & \left(\int_{\xi_{1}}^{\sigma_{1}} \int_{\xi_{2}}^{\sigma_{2}} \int_{\xi_{3}}^{\sigma_{3}}\left|h\left(\delta_{1}, \delta_{2}, \delta_{3}\right)\right|\left|f\left(\delta_{1}, \delta_{2}, \delta_{3}\right)\right|^{p} \diamond \delta_{1} \diamond \delta_{2} \diamond \delta_{3}\right)^{1 / p} \\
& +\left(\int_{\xi_{1}}^{\sigma_{1}} \int_{\xi_{2}}^{\sigma_{2}} \int_{\xi_{3}}^{\sigma_{3}}\left|h\left(\delta_{1}, \delta_{2}, \delta_{3}\right)\right|\left|g\left(\delta_{1}, \delta_{2}, \delta_{3}\right)\right|^{p} \diamond \delta_{1} \diamond \delta_{2} \diamond \delta_{3}\right)^{1 / p} \\
= & M^{1 / p}+N^{1 / p} .
\end{aligned}
$$

From the above inequality, we have

$$
\begin{aligned}
W= & M^{1 / p}+N^{1 / p} \\
= & M^{1 / p-1} \int_{\xi_{1}}^{\sigma_{1}} \int_{\xi_{2}}^{\sigma_{2}} \int_{\xi_{3}}^{\sigma_{3}}\left|h\left(\delta_{1}, \delta_{2}, \delta_{3}\right)\right|\left|f\left(\delta_{1}, \delta_{2}, \delta_{3}\right)\right|^{p} \diamond \delta_{1} \diamond \delta_{2} \diamond \delta_{3} \\
& +N^{1 / p-1} \int_{\xi_{1}}^{\sigma_{1}} \int_{\xi_{2}}^{\sigma_{2}} \int_{\xi_{3}}^{\sigma_{3}}\left|h\left(\delta_{1}, \delta_{2}, \delta_{3}\right)\right|\left|g\left(\delta_{1}, \delta_{2}, \delta_{3}\right)\right|^{p} \diamond \delta_{1} \diamond \delta_{2} \diamond \delta_{3} \\
= & \int_{\xi_{1}}^{\sigma_{1}} \int_{\xi_{2}}^{\sigma_{2}} \int_{\xi_{3}}^{\sigma_{3}}\left|h\left(\delta_{1}, \delta_{2}, \delta_{3}\right)\right|\left(\left|f\left(\delta_{1}, \delta_{2}, \delta_{3}\right)\right|^{p} M^{1 / p-1}\right. \\
& \left.+\left|g\left(\delta_{1}, \delta_{2}, \delta_{3}\right)\right|^{p} N^{1 / p-1}\right) \diamond \delta_{1} \diamond \delta_{2} \diamond \delta_{3} \\
\geq & \int_{\xi_{1}}^{\sigma_{1}} \int_{\xi_{2}}^{\sigma_{2}} \int_{\xi_{3}}^{\sigma_{3}}\left|h\left(\delta_{1}, \delta_{2}, \delta_{3}\right)\right|\left|f\left(\delta_{1}, \delta_{2}, \delta_{3}\right)+g\left(\delta_{1}, \delta_{2}, \delta_{3}\right)\right|^{p} \\
& \times\left(M^{1 / p}+N^{1 / p}\right)^{1-p} \diamond \delta_{1} \diamond \delta_{2} \diamond \delta_{3} \\
= & \int_{\xi_{1}}^{\sigma_{1}} \int_{\xi_{2}}^{\sigma_{2}} \int_{\xi_{3}}^{\sigma_{3}}\left|h\left(\delta_{1}, \delta_{2}, \delta_{3}\right)\right| f\left(\delta_{1}, \delta_{2}, \delta_{3}\right)+\left.g\left(\delta_{1}, \delta_{2}, \delta_{3}\right)\right|^{p} W^{1-p} \diamond \delta_{1} \diamond \delta_{2} \diamond \delta_{3} \\
= & W^{1-p} \int_{\xi_{1}}^{\sigma_{1}} \int_{\xi_{2}}^{\sigma_{2}} \int_{\xi_{3}}^{\sigma_{3}}\left|h\left(\delta_{1}, \delta_{2}, \delta_{3}\right)\right|\left|f\left(\delta_{1}, \delta_{2}, \delta_{3}\right)+g\left(\delta_{1}, \delta_{2}, \delta_{3}\right)\right|^{p} \diamond \delta_{1} \diamond \delta_{2} \diamond \delta_{3} .
\end{aligned}
$$

That is,

$$
W \geq W^{1-p} \int_{\xi_{1}}^{\sigma_{1}} \int_{\xi_{2}}^{\sigma_{2}} \int_{\xi_{3}}^{\sigma_{3}}\left|h\left(\delta_{1}, \delta_{2}, \delta_{3}\right)\right|\left|f\left(\delta_{1}, \delta_{2}, \delta_{3}\right)+g\left(\delta_{1}, \delta_{2}, \delta_{3}\right)\right|^{p} \diamond \delta_{1} \diamond \delta_{2} \diamond \delta_{3} .
$$

Hence, we have

$$
W^{p} \geq \int_{\xi_{1}}^{\sigma_{1}} \int_{\xi_{2}}^{\sigma_{2}} \int_{\xi_{3}}^{\sigma_{3}}\left|h\left(\delta_{1}, \delta_{2}, \delta_{3}\right)\right|\left|f\left(\delta_{1}, \delta_{2}, \delta_{3}\right)+g\left(\delta_{1}, \delta_{2}, \delta_{3}\right)\right|^{p} \diamond \delta_{1} \diamond \delta_{2} \diamond \delta_{3} .
$$

Based on the above inequality, we obtain

$$
\begin{aligned}
& {\left[\left(\int_{\xi_{1}}^{\sigma_{1}} \int_{\xi_{2}}^{\sigma_{2}} \int_{\xi_{3}}^{\sigma_{3}}\left|h\left(\delta_{1}, \delta_{2}, \delta_{3}\right)\right|\left|f\left(\delta_{1}, \delta_{2}, \delta_{3}\right)\right|^{p} \diamond \delta_{1} \diamond \delta_{2} \diamond \delta_{3}\right)^{1 / p}\right.} \\
& \left.\quad+\left(\int_{\xi_{1}}^{\sigma_{1}} \int_{\xi_{2}}^{\sigma_{2}} \int_{\xi_{3}}^{\sigma_{3}}\left|h\left(\delta_{1}, \delta_{2}, \delta_{3}\right)\right|\left|g\left(\delta_{1}, \delta_{2}, \delta_{3}\right)\right|^{p} \diamond \delta_{1} \diamond \delta_{2} \diamond \delta_{3}\right)^{1 / p}\right]^{p} \\
& \quad \geq \int_{\xi_{1}}^{\sigma_{1}} \int_{\xi_{2}}^{\sigma_{2}} \int_{\xi_{3}}^{\sigma_{3}}\left|h\left(\delta_{1}, \delta_{2}, \delta_{3}\right)\right|\left|f\left(\delta_{1}, \delta_{2}, \delta_{3}\right)+g\left(\delta_{1}, \delta_{2}, \delta_{3}\right)\right|^{p} \diamond \delta_{1} \diamond \delta_{2} \diamond \delta_{3} .
\end{aligned}
$$


Through the above inequalities, we finally get the result

$$
\begin{gathered}
\left(\frac{\int_{\xi_{1}}^{\sigma_{1}} \int_{\xi_{2}}^{\sigma_{2}} \int_{\xi_{3}}^{\sigma_{3}}\left|h\left(\delta_{1}, \delta_{2}, \delta_{3}\right)\right|\left|f\left(\delta_{1}, \delta_{2}, \delta_{3}\right)+g\left(\delta_{1}, \delta_{2}, \delta_{3}\right)\right|^{p} \diamond \delta_{1} \diamond \delta_{2} \diamond \delta_{3}}{\int_{\xi_{1}}^{\sigma_{1}} \int_{\xi_{2}}^{\sigma_{2}} \int_{\xi_{3}}^{\sigma_{3}}\left|h\left(\delta_{1}, \delta_{2}, \delta_{3}\right)\right|\left|f\left(\delta_{1}, \delta_{2}, \delta_{3}\right)+g\left(\delta_{1}, \delta_{2}, \delta_{3}\right)\right|^{r} \diamond \delta_{1} \diamond \delta_{2} \diamond \delta_{3}}\right)^{1 /(p-r)} \\
\leq\left(\frac{\int_{\xi_{1}}^{\sigma_{1}} \int_{\xi_{2}}^{\sigma_{2}} \int_{\xi_{3}}^{\sigma_{3}}\left|h\left(\delta_{1}, \delta_{2}, \delta_{3}\right)\right|\left|f\left(\delta_{1}, \delta_{2}, \delta_{3}\right)\right|^{p} \diamond \delta_{1} \diamond \delta_{2} \diamond \delta_{3}}{\int_{\xi_{1}}^{\sigma_{1}} \int_{\xi_{2}}^{\sigma_{2}} \int_{\xi_{3}}^{\sigma_{3}}\left|h\left(\delta_{1}, \delta_{2}, \delta_{3}\right)\right|\left|f\left(\delta_{1}, \delta_{2}, \delta_{3}\right)\right|^{r} \diamond \delta_{1} \diamond \delta_{2} \diamond \delta_{3}}\right)^{1 /(p-r)} \\
\quad+\left(\frac{\int_{\xi_{1}}^{\sigma_{1}} \int_{\xi_{2}}^{\sigma_{2}} \int_{\xi_{3}}^{\sigma_{3}}\left|h\left(\delta_{1}, \delta_{2}, \delta_{3}\right)\right|\left|g\left(\delta_{1}, \delta_{2}, \delta_{3}\right)\right|^{p} \diamond \delta_{1} \diamond \delta_{2} \diamond \delta_{3}}{\int_{\xi_{1}}^{\sigma_{1}} \int_{\xi_{2}}^{\sigma_{2}} \int_{\xi_{3}}^{\sigma_{3}}\left|h\left(\delta_{1}, \delta_{2}, \delta_{3}\right)\right|\left|g\left(\delta_{1}, \delta_{2}, \delta_{3}\right)\right|^{r} \diamond \delta_{1} \diamond \delta_{2} \diamond \delta_{3}}\right)^{1 /(p-r)} .
\end{gathered}
$$

Thus, Theorem 4.8 is completely proved.

Theorem 4.9 Let $f, g, h: \mathbb{T} \rightarrow \mathbb{R}$ and $0<r<1<p$.Iff, $g$ and $h$ are $\diamond$-integrable on $[\xi, \sigma]_{\mathbb{T}}$, then we have the following assertion

$$
\begin{aligned}
& \left(\frac{\int_{\xi_{1}}^{\sigma_{1}} \int_{\xi_{2}}^{\sigma_{2}} \cdots \int_{\xi_{n}}^{\sigma_{n}}\left|h\left(\delta_{1}, \delta_{2}, \ldots, \delta_{n}\right)\right| f\left(\delta_{1}, \delta_{2}, \ldots, \delta_{n}\right)+\left.g\left(x_{1}, x_{2} \cdots, x_{n}\right)\right|^{p} \diamond \delta_{1} \oslash \delta_{2} \cdots \diamond \delta_{n}}{\int_{\xi_{1}}^{\sigma_{1}} \int_{\xi_{2}}^{\sigma_{2}} \cdots \int_{\xi_{n}}^{\sigma_{n}}\left|h\left(\delta_{1}, \delta_{2}, \ldots, \delta_{n}\right)\right|\left|f\left(\delta_{1}, \delta_{2}, \ldots, \delta_{n}\right)+g\left(\delta_{1}, \delta_{2}, \ldots, \delta_{n}\right)\right|^{r} \oslash \delta_{1} \diamond \delta_{2} \cdots \diamond \delta_{n}}\right)^{1 /(p-r)} \\
& \leq\left(\frac{\int_{\xi_{1}}^{\sigma_{1}} \int_{\xi_{2}}^{\sigma_{2}} \cdots \int_{\xi_{n}}^{\sigma_{n}}\left|h\left(\delta_{1}, \delta_{2}, \ldots, \delta_{n}\right)\right|\left|f\left(\delta_{1}, \delta_{2}, \ldots, \delta_{n}\right)\right|^{p} \diamond \delta_{1} \diamond \delta_{2} \cdots \diamond \delta_{n}}{\left.\int_{\xi_{1}}^{\sigma_{1}} \int_{\xi_{2}}^{\sigma_{2}} \cdots \int_{\xi_{n}}^{\sigma_{n}}\left|h\left(\delta_{1}, \delta_{2}, \ldots, \delta_{n}\right)\right| f\left(\delta_{1}, \delta_{2}, \ldots, \delta_{n}\right)\right|^{r} \diamond \delta_{1} \diamond \delta_{2} \cdots \diamond \delta_{n}}\right)^{1 /(p-r)} \\
& +\left(\frac{\int_{\xi_{1}}^{\sigma_{1}} \int_{\xi_{2}}^{\sigma_{2}} \cdots \int_{\xi_{n}}^{\sigma_{n}}\left|h\left(\delta_{1}, \delta_{2}, \ldots, \delta_{n}\right)\right|\left|g\left(\delta_{1}, \delta_{2}, \ldots, \delta_{n}\right)\right|^{p} \diamond \delta_{1} \diamond \delta_{2} \cdots \diamond \delta_{n}}{\left.\int_{\xi_{1}}^{\sigma_{1}} \int_{\xi_{2}}^{\sigma_{2}} \cdots \int_{\xi_{n}}^{\sigma_{n}}\left|h\left(\delta_{1}, \delta_{2}, \ldots, \delta_{n}\right)\right| g\left(\delta_{1}, \delta_{2}, \ldots, \delta_{n}\right)\right|^{r} \diamond \delta_{1} \diamond \delta_{2} \cdots \diamond \delta_{n}}\right)^{1 /(p-r)} .
\end{aligned}
$$

Proof Similar to the proof of Theorem 4.8, we get the result of Theorem 4.9.

Remark 4.10 For the inequality of Theorem 3.7 in Ref. [17], we generalize it in this paper and obtain the generalized inequalities in Theorem 4.8 and Theorem 4.9.

Acknowledgements

The authors gratefully acknowledge the anonymous referees for their constructive comments and advice on the earlier version for this paper.

Funding

This paper was supported by the Fundamental Research Funds for the Central Universities (Nos. 2019MS129, 2015ZD29).

\section{Competing interests}

The authors declare that they have no competing interests.

Authors' contributions

The authors read and approved the final manuscript.

\section{Author details}

${ }^{1}$ North China Electric Power University, Baoding, P.R. China. ${ }^{2}$ College of Science and Technology, North China Electric Power University, Baoding, P.R. China.

\section{Publisher's Note}

Springer Nature remains neutral with regard to jurisdictional claims in published maps and institutional affiliations.

Received: 27 August 2019 Accepted: 12 December 2019 Published online: 19 December 2019

\section{References}

1. Ozkan, U.M., Sarikaya, M.Z., Yildirim, H.: Extensions of certain integral inequalities on time scales. Appl. Math. Lett. 21 993-1000 (2008)

2. Hilger, S.: Ein Maßkettenkalkül mit Anwendung auf Zentrumsmannigfaltigkeiten. Ph.D. thesis, Universität Würzburg, Würzburg, Germany (1988)

3. Agarwal, R.P., O'Regan, D., Saker, S.: Dynamic Inequalities on Time Scales. Springer, New York (2014) 
4. Agarwal, R.P., Bohner, M., Peterson, A.: Inequalities on time scales: a survey. Math. Inequal. Appl. 4, 535-557 (2001)

5. Wang, M.K., Li, Y.M., Chu, Y.M.: Inequalities and infinite product formula for Ramanujan generalized modular equation function. Ramanujan J. 46(1), 189-200 (2018)

6. Yang, Z.-H., Tian, J.-F.: A class of completely mixed monotonic functions involving the gamma function with applications. Proc. Am. Math. Soc. 146(11), 4707-4721 (2018)

7. Tian, J.: Note on common fixed point theorems in fuzzy metric spaces using the CLRg property. Fuzzy Sets Syst. 379, 134-137 (2020)

8. Wang, M.K., Zhang, W., Chu, Y.M.: Monotonicity, convexity and inequalities involving the generalized elliptic integrals. Acta Math. Sci. Ser. B Engl. Ed. 39(5), 1440-1450 (2019)

9. Tian, J.F., Ha, M.H., Wang, C.: Improvements of generalized Hölder's inequalities and their applications. J. Math. Inequal. 12, 459-471 (2018)

10. Chen, G.S., Huang, F.L., Liao, L.F.: Generalizations of Hölder inequality and some related results on time scales. J. Inequal. Appl. 2014, 207 (2014)

11. Agahi, H., Ouyang, Y., Mesiar, R., Pap, E., Štrboja, M.: Hölder and Minkowski type inequalities for pseudo-integral. Appl. Math. Comput. 217, 8630-8639 (2011)

12. Tian, J.F., Ha, M.H.: Properties of generalized sharp Hölder's inequalities. J. Math. Inequal. 11, 511-525 (2017)

13. Zhao, C., Cheung, W.: Hölder's reverse inequality and its applications. Publ. L. Math. 99, 211-216 (2016)

14. Tian, J.F: New property of a generalized Hölder's inequality and its applications. Inf. Sci. 288, 45-54 (2014)

15. Tian, J.F.: Reversed version of a generalized sharp Hölder's inequality and its applications. Inf. Sci. 201, 61-69 (2012)

16. Lou, Y.T.: Generalizations of Young inequality and Hölder inequality. J. Nanjing Inst. Posts Telecommun. 14, 98-103 (1994)

17. Chen, G.S., Wei, C.D.: The diamond integral reverse Hölder inequality and related results on time scales. Adv. Differ. Equ. 2015,180 (2015)

18. Tian, J.F: Triple Diamond-Alpha integral and Hölder-type inequalities. J. Inequal. Appl. 2018, 111 (2018)

19. Tian, J.F., Zhu, Y.R., Cheung, W.S.: N-tuple Diamond-Alpha integral and inequalities on time scales. Rev. R. Acad. Cienc. Exactas Fís. Nat., Ser. A Mat. 113, 2189-2200 (2019)

\section{Submit your manuscript to a SpringerOpen ${ }^{\circ}$ journal and benefit from:}

- Convenient online submission

- Rigorous peer review

- Open access: articles freely available online

- High visibility within the field

- Retaining the copyright to your article

Submit your next manuscript at $\boldsymbol{\nabla}$ springeropen.com 